\title{
Computational Modelling of Structural, Electronic, Optical and Vibrational Properties of PVK/C 60 Nanoheterostructure Interfaces
}

\author{
Van Wellington Elloh ${ }^{1,2}$, Abu Yaya ${ }^{1 *} \mathbb{D}$, Abhishek Kumar Mishra ${ }^{2}$, Gebremedhn Gebreyesus ${ }^{3}$, Kwabena \\ Kan-Dapaah ${ }^{4}$, Eric. Kwabene Kyeh Abavare ${ }^{5}$, Boateng Onwona-Agyeman ${ }^{1}$ \\ 1 Department of Materials Science and Engineering, School of Engineering Science, CBAS, University of Ghana, Legon, \\ Ghana \\ 2 Department of Physics, University of Petroleum and Energy Studies (UPES), Dehradun, India \\ 3 Department of Physics, School of Physical Science, CBAS, University of Ghana, Legon, Ghana \\ 4 Department of Biomedical Engineering, School of Engineering Science, CBAS, University of Ghana, Legon, Ghana \\ 5 Department of Physics, KNUST, Kumasi, Ghana \\ * Correspondence: ayaya@ug.edu.gh;
}

Scopus Author ID 36674204900

Received: 1.09.2020; Revised: 7.11.2020; Accepted: 10.11.2020; Published: 15.11.2020

\begin{abstract}
In this work, we present first-principles density functional studies of the dynamical properties of three nanoheterostructure interfaces of poly(9-vinylcarbazole)/fullerene $\left(\mathrm{PVK} / \mathrm{C}_{60}\right)$ : $\mathrm{PVK} / \mathrm{C}_{60}(\alpha)$, $\mathrm{PVK} / \mathrm{C}_{60}(\beta)$, and $\mathrm{PVK} / \mathrm{C}_{60}(\gamma)$. Linear response within density functional perturbation theory (DFPT), as implemented in the Quantum Espresso code, has been employed to explore the nature of permittivity, Born effective charges, polarizabilities, and vibrational frequency modes. The vibrational frequencies for the three nanoheterostructure interfaces were computed and their modes assigned. The phonon modes were also classified, and we showed that some important frequency modes are associated with $\mathrm{C}-\mathrm{C}, \mathrm{CH}_{2}$, and $\mathrm{C}-\mathrm{N}$ stretching modes, among others. In addition, computed values of polarizability and permittivity for the nanoheterostructures were seen to have comparable values to that of literature for conjugated polymers. Conspicuous differing characteristics were noticed in the computed infrared and Raman absorption spectra for the three nanoheterostructures, which was due to the structural differences arising from their different stacking patterns. It is noted that the presence of the nitrogen atom plays a significant role in determining their dynamical lattice properties. The permittivity and polarizability tensors of $\mathrm{PVK} / \mathrm{C}_{60}(\alpha), \mathrm{PVK} / \mathrm{C}_{60}(\beta)$, and $\mathrm{PVK} / \mathrm{C}_{60}(\gamma)$ nanoheterostructures were found to be strongly anisotropic.
\end{abstract}

Keywords: PVK/C 60 ; DFT; GGA-PBE; Electronic properties; Dynamical properties.

(C) 2020 by the authors. This article is an open-access article distributed under the terms and conditions of the Creative Commons Attribution (CC BY) license (https://creativecommons.org/licenses/by/4.0/).

\section{Introduction}

In recent times conjugated polymers have drawn appreciable attention in basic and applied research due to the appreciable level of conductivity in doped polymers $[1,2]$. Moreover, a substantial amount of scientific efforts and resources have been focused on gaining a thorough insight into the family of conjugated polymers' electronic and dynamical properties. Fortunately, these materials are handy to synthesize, and above all, they possess suitable environmental and thermal stability [3]. Additionally, these materials possess high formability rendering them pliable in device fabrication because it presents the possibility of different doping levels, which permit the opportunity to tune their electrical and optical properties. 
Presumably, the myriad range of controllable optoelectronic properties, coupled with high stability, render them promising potential applications in biomechanics, nanoelectronics, nanoelectromechanical systems (NEMS), spintronics, optoelectronics, and photonic devices manufacturing industry in addition to biosensors [4], batteries [5] and field effect transistors [6-8]. Essentially, both experimental and theoretical investigations of the dynamical properties of conjugated polymers play a leading role in appreciating the structural and microscopic optoelectronic properties of the materials in various phases in the pristine and in the doped states $[9,10]$. Unfortunately, it is often the case that information obtained by diverse techniques on these conjugated polymers has rarely been consistent [11]. Even though there have been numerous studies of the electronic and dynamical properties of conjugated polymers, a consensus on the vibrational modes' assignment is absent in the literature.

It was found that experimental infrared mode frequencies have been published for some classes of conjugated polymers [12-14]. However, these sources failed to assign most of the observed modes [13], with only one assigning the fundamental modes [14]. In Ref. [15], using a harmonic potential with parameters transferred from other molecules, the vibrational modes of a number of conjugated polymers have been calculated, which appear to be in good agreement with previously published data. Besides these results, there is a lack of comprehensive studies concerning the materials' dynamical properties, notably the dynamical anisotropy. Admissibly, first principles calculations are the sure route to these crossover domains, but they are computationally excessively huge and demanding. Consequently, the calculations of dynamical properties are often the average values calculated from the trace of the dynamical tensors or the arithmetic average of the three principal components of the tensors $[16,17]$, while ignoring the anisotropy information. Notwithstanding, some level of progress has been attained in grasping the anisotropy of the polarizability for a handful of molecules and some scanty molecular crystals [18-21]. Likewise, the same is true for some of the routine molecules [22]. An ab initio dynamics study has been carried out on the pristine poly-paraphenylene vinylene (PPV) polymer, recently revealing some novel properties of these conjugated polymers [23].

In this study, we present the results of first-principles investigation of the dynamical properties of the conducting polymer nanoheterostructure interfaces, $\mathrm{PVK} / \mathrm{C}_{60}(\alpha), \mathrm{PVK} / \mathrm{C}_{60}(\beta)$, and $\mathrm{PVK} / \mathrm{C}_{60}(\gamma)$. First of all, we examined various ground state electronic properties of $\mathrm{PVK} / \mathrm{C}_{60}$ nanoheterostructures, followed by the vibrational properties of these conducting nanoheterostructure interfaces. Furthermore, the calculations of electric field response and Born effective charges were explored to gain insight into other related properties of these nanoheterostructures.

\section{Materials and Methods}

\subsection{Computational technique.}

An ab initio calculation within the density functional theory (DFT) formalism using plane-wave basis sets implemented in Quantum Espresso code [24,25] is employed to model new classes of $\mathrm{PVK} / \mathrm{C}_{60}$ nanoheterostructures. The generalized gradient approximation in the Perdew, Burke, and Ernzerhof (GGA-PBE) scheme and the Grimme van der Waals (vdW) dispersion correction to account for the adverse effects of dispersion forces as proposed by Tkatchenko and Scheffler (TS method). We used the ultrasoft pseudopotentials [26] in describing the interactions between the ions and electrons with Perdew-Burke-Ernzerhof (PBE) 
exchange-correlation functional [27]. The Methfessel-Paxton smearing technique was used for smearing the occupation numbers with Gaussian broadening of up to 0.001 Ry [28]. A plane wave energy cutoff of 30 Ryd is used after testing with periodic boundary conditions applied. Our supercell was oriented along with the z-axis and unit cell geometry with a vacuum space of $15 \AA$ thick in both $\mathrm{x}$ - and y-directions were allowed to ensure negligible interactions between the supercell and its periodic images. The structure was optimized with Broyden-Fletcher Goldfarb-Shanno (BFGS) method [29]. Next, we sampled with a $\Gamma$-centred 5x1x1 MonkhorstPack scheme [30] and a k-point grid followed by fine 9x1x1 grids non-self-consistent field calculations. The latter was carried out to determine quantized energy levels and Fermi energy for band structure calculations in the Gamma-Z direction along the supercell axis, charge density analysis, and the density of electronic states (DOS), and partial density of electronic states (PDOS) computations.

Density functional perturbation theory (DFPT) calculations were employed in infrared intensity and Raman vibrational mode analysis. Since during the time of our calculations, the phonon code with ultrasoft pseudopotential (USPP) is not yet available. The third-order derivatives for norm-conserving pseudopotential (NCPP) are not yet implemented with GGA, we used the norm-conserving pseudopotential (NCPP) and the local density approximation (LDA) in our first principles Raman and infrared spectral calculations and analysis. Calculations and analysis of states' phonon density (DOS) and phonon projected density of state (PDOS) were equally carried out. We performed a self-consistent field ground-state calculation for the equilibrium structure molecule using the standard plane-wave program. Optical absorption spectra are computed by time-dependent density functional perturbation theory (TDDFPT) using the Liouville-Lanczos approach [31,32]. In this case, the k-point sampling is restricted to $\Gamma$-point only.

\section{Results and Discussion}

\subsection{Structural properties of the nanoheterostructures.}

Figure 1 illustrates the structures and different configurations of the nanoheterostructures investigated in this work. As shown in figure $1(\alpha)$, the five-membered ring of $\mathrm{C}_{60}$, and the $\pi$-conjugate plane of PVK is modeled considering a parallel configuration. The six-membered ring of $\mathrm{C}_{60}$ and the $\pi$-conjugate plane of PVK modeled in a parallel arrangement is also shown in figure $1(\beta)$. In the third configuration, the six-membered ring of $\mathrm{C}_{60}$ is constructed to face the nitrogen-containing ring of PVK, Figure $1(\gamma)$.

The structural stability of the PVK/C60 nanoheterostructures are evaluated by calculating the formation energy for each of the described configurations to determine the most energetically favorable stacking structure using equation (1) below [33]:

$$
\Delta \mathrm{E}=\mathrm{E}_{\mathrm{CHN}}-\mathrm{N}_{\mathrm{C}} \mathrm{E}_{\mathrm{C}}-\mathrm{N}_{\mathrm{H}} \mathrm{E}_{\mathrm{H}}-\mathrm{N}_{\mathrm{N}} \mathrm{E}_{\mathrm{N}}
$$

where $E_{C H N}$ is the total energy of the ground state of the corresponding nanoheterostructure modeled. $\mathrm{E}_{\mathrm{C}}$ is the total energy of carbon in its ground state (graphite), $\mathrm{N}_{\mathrm{C}}$ is the number of carbon atoms in the nanoheterostructure. $\mathrm{E}_{\mathrm{H}}$ is the total energy of the ground state of $\mathrm{H}, \mathrm{N}_{\mathrm{H}}$ is the number of hydrogen atoms, $E_{N}$ is the total energy of the ground state of $\mathrm{N}$ and $\mathrm{N}_{\mathrm{N}}$ is the number of nitrogen atoms in the nanoheterostructure modeled.

The calculated formation energies per atom for each of the nanoheterostructures is tabulated in Table 1. It is observed that all of the new nanoheterostructure modeled have 
negative formation energies per atom, indicating that these new structures are thermodynamically stable.

To gain further insight into the new structures, we calculate the formation energy per atom for $\mathrm{C}_{60}$ and the formation energy per atom for PVK separately and found their values to be $-5.263 \mathrm{eV}$ and $-6.486 \mathrm{eV}$, respectively, which are much larger than the formation energies per atom in Table 1 for each of the newly designed nanoheterostructures in this present study. This shows that the new nanoheterostructures are more stable and thermodynamically favorable in comparison to the individual compounds.

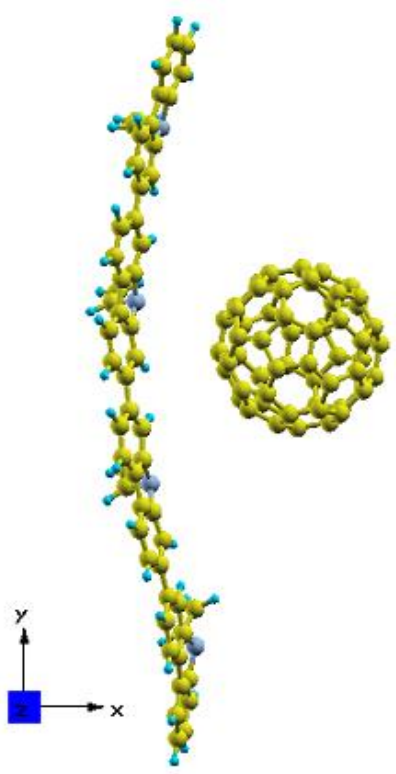

$(\alpha)$

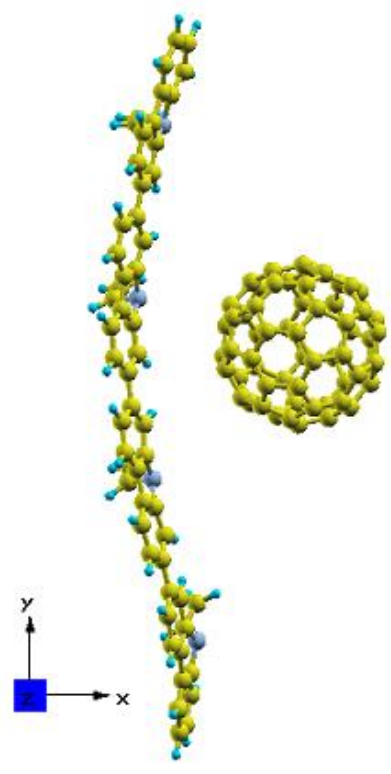

( $\beta)$

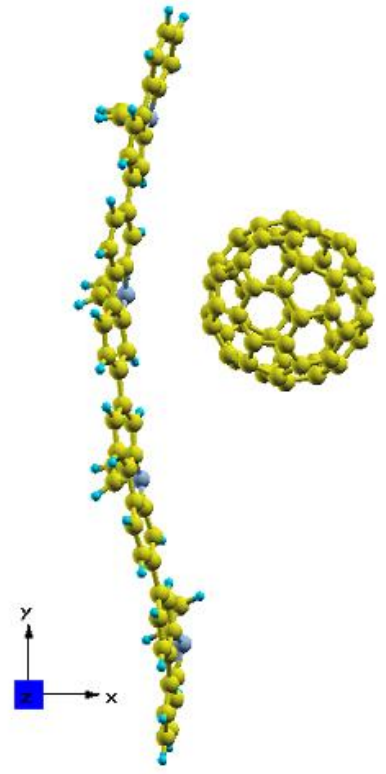

$(\gamma)$

Figure 1. Schematic illustration of different stacking patterns of the $\mathrm{PVK} / \mathrm{C}_{60}$ nanoheterostructure composite models for: $(\alpha)$ a five-membered ring of $\mathrm{C}_{60}$ and the $\pi$-conjugate plane of PVK arranged in parallel, $(\beta)$ a sixmembered ring of $\mathrm{C}_{60}$ and the $\pi$-conjugate plane of PVK arranged in parallel, $(\gamma)$ six-membered ring of $\mathrm{C}_{60}$ facing the nitrogen-containing ring of PVK. A separation distance of $\sim 3.38 \AA$ was kept between PVK and $\mathrm{C}_{60}$ in all cases throughout.

Table 1. Formation Energy Per Atom Calculated Using Equation 1.

\begin{tabular}{l|l} 
Model type & Formation Energy $(\mathbf{e V})$ \\
\hline $\mathrm{PVK} / \mathrm{C}_{60}(\boldsymbol{\alpha})$ & -8.106 \\
\hline $\mathrm{PVK} / \mathrm{C}_{60}(\boldsymbol{\beta})$ & -8.492 \\
\hline $\mathrm{PVK} / \mathrm{C}_{60}(\boldsymbol{\gamma})$ & -9.114
\end{tabular}

Furthermore, our calculations reveal that in the nanoheterostructure model in which the six-membered ring of $\mathrm{C}_{60}$ faces the nitrogen-containing ring of $\mathrm{PVK}, \mathrm{PVK} / \mathrm{C}_{60}(\gamma)$, is the most favored and the most stable as compared to the other two configurations in which the fivemembered ring of $\mathrm{C}_{60}$ and the $\pi$-conjugate plane of PVK are arranged in parallel and the case in which the six-membered ring of $\mathrm{C}_{60}$ and the $\pi$-conjugate plane of $\mathrm{PVK}$ are arranged in parallel, $\mathrm{PVK} / \mathrm{C}_{60}(\alpha)$ and $\mathrm{PVK} / \mathrm{C}_{60}(\beta)$ respectively.

As a result of the stacking patterns in the $\mathrm{PVK} / \mathrm{C}_{60}(\alpha), \mathrm{PVK} / \mathrm{C}_{60}(\beta)$, and $\mathrm{PVK} / \mathrm{C}_{60}(\gamma)$ configurations, the three structures are found to shrink accordingly as shown in table 2 . The values of the energy differences $\mathrm{E}_{0}(\mathrm{eV})$ of the simulated nanoheterostructures show progressive stability from stable to most stable nanoheterostructure, respectively. 
Table 2. Optimized Lattice Parameters a $(\AA), b(\AA), c(\AA)$, Energy gap Eg(eV), energy difference $E_{0}(e V)$ of different nanoheterostructures.

\begin{tabular}{l|l|l|l|l|l} 
Model type & $\mathbf{a}(\AA)$ & $\mathbf{b}(\AA)$ & $\mathbf{c}(\AA)$ & $\mathbf{E}(\mathbf{A})$ & $\mathbf{E})(\mathbf{e V})$ \\
\hline $\mathrm{PVK} / \mathrm{C}_{60}(\boldsymbol{\alpha})$ & 12.328 & 9.350 & 9.458 & 1.276 & 46.704 \\
\hline $\mathrm{PVK} / \mathrm{C}_{60}(\boldsymbol{\beta})$ & 9.277 & 11.505 & 9.294 & 1.117 & 6.192 \\
\hline $\mathrm{PVK} / \mathrm{C}_{60}(\boldsymbol{\gamma})$ & 9.131 & 12.113 & 9.067 & 0.893 & 0.000
\end{tabular}

Among the three nanoheterostructure configurations investigated, it is observed that the $\mathrm{C}-\mathrm{C}$ bonds and $\mathrm{C}-\mathrm{N}$ bonds exhibit slightly smaller bond lengths in the $\mathrm{PVK} / \mathrm{C}_{60}(\alpha)$ and $\mathrm{PVK} / \mathrm{C}_{60}(\gamma)$ configurations with respective to the bond lengths of $1.406 \AA$, $1.403 \AA$, and 1.392 $\AA, 1.393 \AA$ as compare to that in the $\mathrm{PVK} / \mathrm{C}_{60}(\beta)$ configuration having bond lengths of 1.408 $\AA$ and $1.394 \AA$, (see table 3).

Table 3. Optimized N-C, C-C \& C-H Bond Lengths $(\AA)$ of the Modelled Nanoheterostructures.

\begin{tabular}{l|c|c|c}
\multirow{2}{*}{ Model type } & \multicolumn{3}{|c}{ Bond Length $(\mathbf{A})$} \\
\cline { 2 - 4 } & $\mathbf{N}-\mathbf{C}$ & $\mathbf{C}-\mathbf{C}$ & $\mathbf{C}-\mathbf{H}$ \\
\hline $\mathrm{PVK} / \mathrm{C}_{60}(\boldsymbol{\alpha})$ & 1.406 & 1.392 & 1.085 \\
\hline $\mathrm{PVK} / \mathrm{C}_{60}(\boldsymbol{\beta})$ & 1.408 & 1.394 & 1.086 \\
\hline $\mathrm{PVK} / \mathrm{C}_{60}(\boldsymbol{\gamma})$ & 1.403 & 1.393 & 1.086
\end{tabular}

This indicates stronger intra-chain interactions in these two nanoheterostructure configurations. In fact, shorter bond lengths and consequently stronger bonds are found to be indicative of more stability of the nanoheterostructures. As a result, $\mathrm{N}-\mathrm{C}$ bonds are found to be more stiffer in $\mathrm{PVK} / \mathrm{C}_{60}(\alpha)$ and $\mathrm{PVK} / \mathrm{C}_{60}(\gamma)$ nanoheterostructures than in the $\mathrm{PVK} / \mathrm{C}_{60}(\beta)$ nanoheterostructure, which gives rise to their stability and rigid nature.

Relative to the adsorption distance of $3.383 \AA$, we calculate the adsorption energies, $E_{a d}$, for the PVK/C60 nanoheterostructures to be $-2.235,-1.485$, and $-1.003 \mathrm{eV}$ respectively. The adsorption energies, $E_{a d}$, are determined from the relation:

$$
E_{a d}=\left(E_{P V K / C_{60}}-E_{P V K}-E_{C_{60}}\right)
$$

where $E_{P V K / C_{60}}, E_{P V K}, E_{C_{60}}$ represent the total ground-state energy of the PVK/C 60 composite, the total energy of PVK, the total energy of $\mathrm{C}_{60}$, respectively. Quantum Espresso code reports the value of dispersion contribution to total energy for every step during the geometry optimization process. Accordingly, the dispersion contribution to adsorption energy is determined using Equation 2 above by replacing the terms on the right-hand side with the dispersion contribution quantities for each structure's converged geometry.

\subsection{Electronic properties of the nanoheterostructures.}

The electronic band structures at high-symmetry k-points for $\mathrm{PVK} / \mathrm{C}_{60}(\alpha), \mathrm{PVK} / \mathrm{C}_{60}(\beta)$, and $\mathrm{PVK} / \mathrm{C}_{60}(\gamma)$ nanoheterostructures are shown in Figure 2. The calculated band gaps for the different PVK/C60 nanoheterostructure stacking, Figures 2 ( $\mathrm{a}, \mathrm{b} \& \mathrm{c}$ ), are $1.276 \mathrm{eV}, 1.117 \mathrm{eV}$, and $0.893 \mathrm{eV}$, respectively.

Since GGA calculations tend to underestimate the energy band-gap of materials, there is a possibility that our currently calculated band-gap energies may not be exact in comparison to the experimental value. However, this difference may not be too significant, but the origin could be attributed to the DFT formalism's inherent problem. The band-gap occurs due to mixed hybridization of $\mathrm{N}$ and $\mathrm{H}$ 's valence states with that of $\mathrm{C}$ atoms in the respective nanoheterostructures. The calculated band gap values, along with the optimized lattice parameters and other structural values of all three model types, are presented in Table 2. 
Upon further analysis of the electronic band structure plots, Figures $2(a, b \& c)$, it is found out that these nanoheterostructures are narrow and direct band-gap materials with bandgap values of $1.276,1.117$, and $0.893 \mathrm{eV}$, with the valance band and conduction band located at the high-symmetry k-points. This makes it a good candidate for photo-physical applications because it is a preferred material for the fabrication of optoelectronic devices. These new materials have tunable band-gap and may have applications in various fields and are promising candidates to facilitate band-gap engineering development for applications in infrared optoelectronics, nanoelectronics, and nano-optics. By matching the band structures of $\mathrm{C}_{60}$ and PVK with the newly modeled nanoheterostructures it is revealed that the new bands are formed due to the hybridization of the N-p and C-p orbital states in the new proposed nanoheterostructures.

(a)

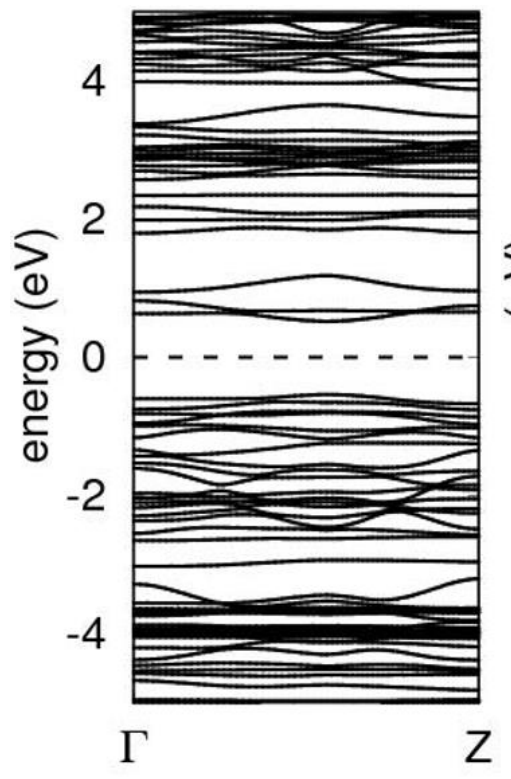

(b)

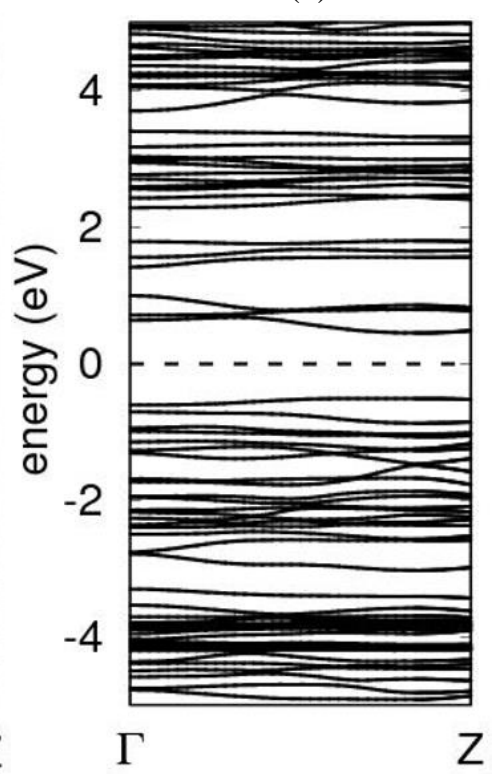

(c)

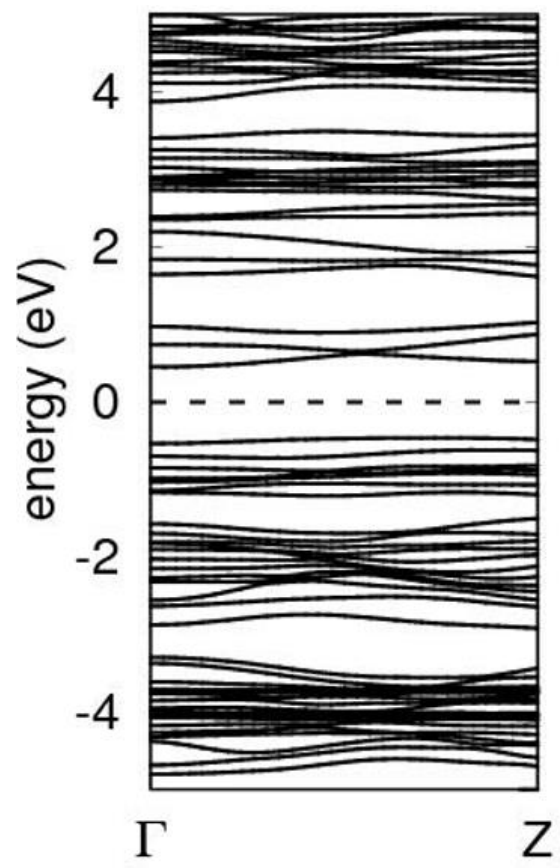

Figure 2. Calculated band structures at high-symmetry k-points of the $\mathrm{PVK} / \mathrm{C}_{60}$ nanoheterostructure interfaces with fermi energy level set at zero for: (a) $\mathrm{PVK} / \mathrm{C}_{60}(\alpha)$, (b) $\mathrm{PVK} / \mathrm{C}_{60}(\beta)$, (c) $\mathrm{PVK} / \mathrm{C}_{60}(\gamma)$ nanoheterostructure interfaces respectively. 
We further analyze the nature of interactions and origin of energy gaps in these nanoheterostructures by way of plotting the electronic DOS, total DOS (t-DOS), and partial DOS (p-DOS) of the individual $\mathrm{PVK} / \mathrm{C}_{60}$ nanoheterostructures in Figures 3 , 4, and 5 respectively. For comparison, the Fermi energy, EF, the level was set as zero on the energy scale of the energy band-gap, Eg, between the occupied atomic orbitals and the empty atomic bands and the same for the DOS, which is shown in Figures 3, 4 and 5. The total and partial electron density of states (t-DOS and p-DOS) plainly reveal that the portion of DOS below -4 $\mathrm{eV}$ is due to $\mathrm{N}$ and $\mathrm{C}$ atoms even though a larger proportion of the contributions is coming from $\mathrm{N}$ atoms only. The central portion, which is above $-4 \mathrm{eV}$, is due to the contributions from $\mathrm{N}$ and $\mathrm{C}$ atoms and the contribution of $\mathrm{H}$ atoms. Figures 3 and 4 show only positive contributions to the total and partial density of electrons' states for the PVK/C60 nanoheterostructure stackings.

(a)

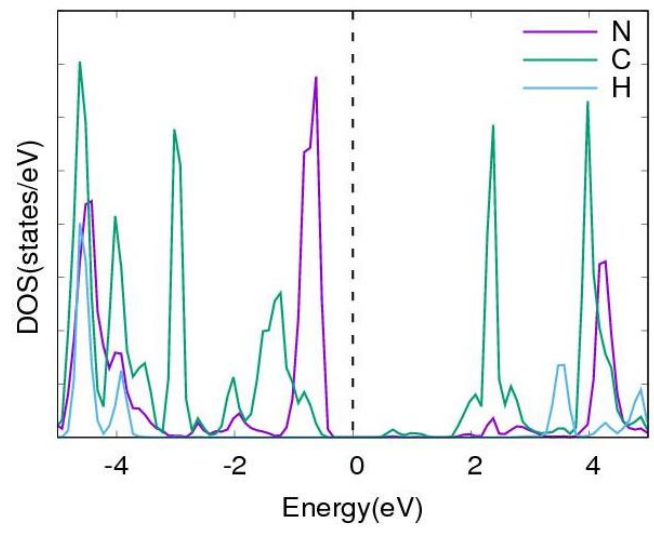

(b)

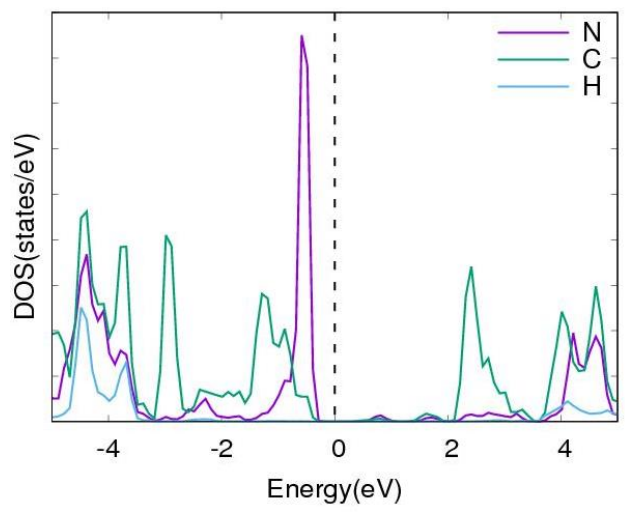

(c)

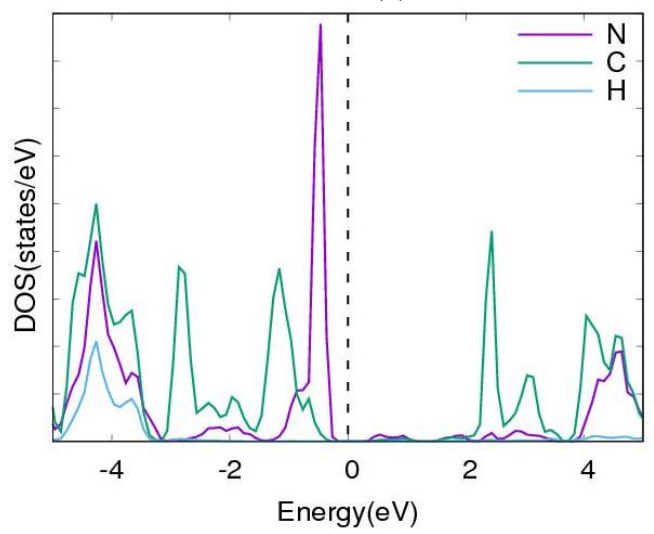

Figure 3. Electronic density of states (DOS) for the $\mathrm{PVK} / \mathrm{C}_{60}$ nanoheterostructure interface with fermi energy level set at zero for (a) $\mathrm{PVK} / \mathrm{C}_{60}(\boldsymbol{\alpha})$, (b) $\mathrm{PVK} / \mathrm{C}_{60}(\boldsymbol{\beta})$, (c) $\mathrm{PVK} / \mathrm{C}_{60}(\boldsymbol{\gamma})$ nanoheterostructure interfaces respectively.

Upon the visualization of the atomic orbitals, we note that the DOS near the energy gaps is essential of $\mathrm{p}_{z}$ electronic character manifestations of the anti-bonding and bonding of $\mathrm{N}$ atoms hybridizing with $\mathrm{p}_{z}$ states of carbon atoms. From the p-DOS shown in Figure 5, it can be seen that the s- and p-orbitals of carbon and also p-orbitals of nitrogen are the dominant class of contributors to the valence bands and conduction band, i.e., both tops of filled bands and the bottom of unoccupied bands are influenced by s- and p-orbital states of carbon and nitrogen atoms resulting in the non-covalent $\pi$-stacking in $\mathrm{PVK}_{6} \mathrm{C}_{60}$ nanoheterostructure stackings as seen in Figures 1(a, b \& c). 
(a)

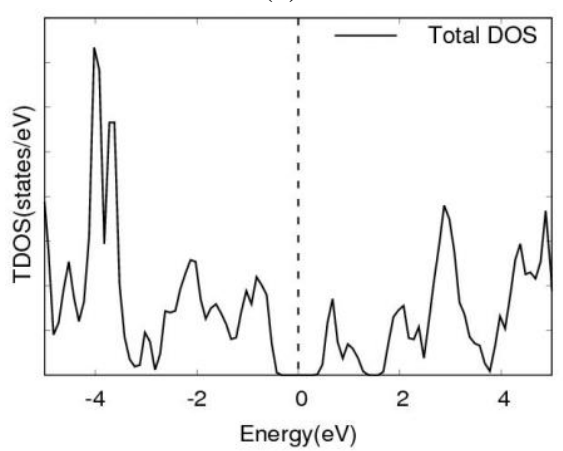

(b)

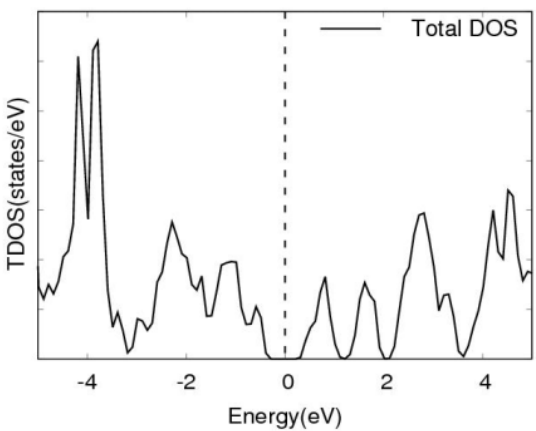

(c)

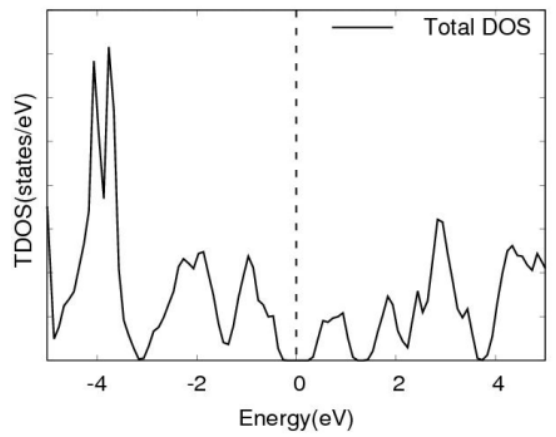

Figure 4. Electronic density of states (t-DOS) for the $\mathrm{PVK} / \mathrm{C}_{60}$ nanoheterostructure interface with fermi energy level set at zero for (a) $\mathrm{PVK} / \mathrm{C}_{60}(\alpha)$, (b) $\mathrm{PVK} / \mathrm{C}_{60}(\beta)$, and (c) $\mathrm{PVK} / \mathrm{C}_{60}(\gamma)$ nanoheterostructure interfaces respectively.

(a)

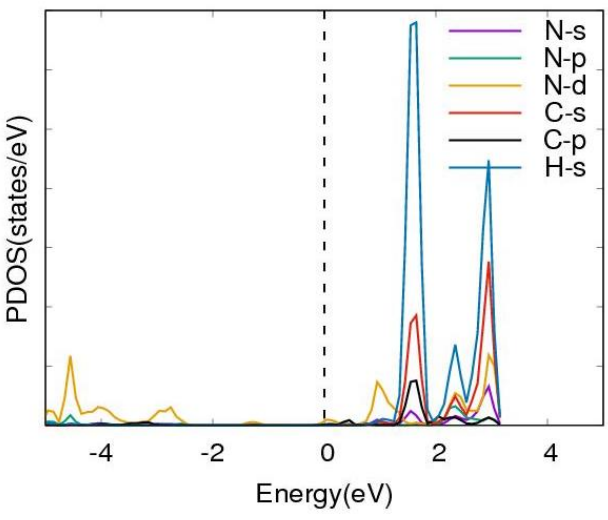

(b)

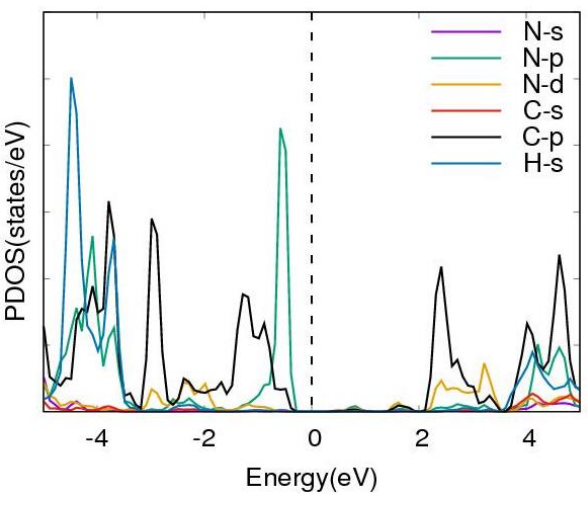

(c)

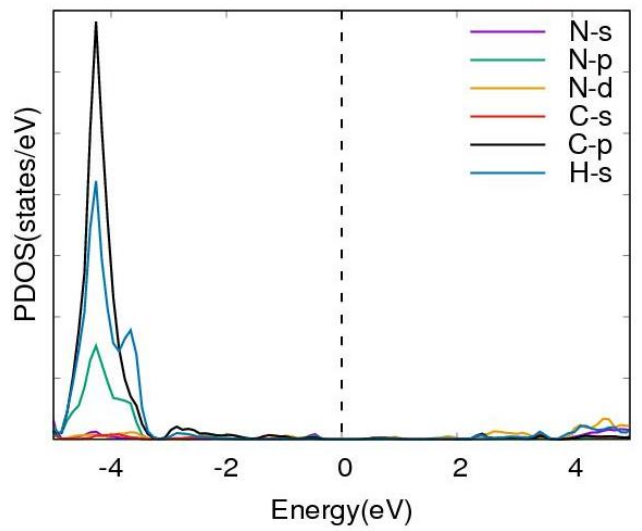

Figure 5. Partial density of states (p-DOS) for the $\mathrm{PVK} / \mathrm{C}_{60}$ nanoheterostructure interface with fermi energy level set at zero for (a) $\mathrm{PVK} / \mathrm{C}_{60}(\alpha)$, (b) $\mathrm{PVK} / \mathrm{C}_{60}(\beta)$, and (c) $\mathrm{PVK} / \mathrm{C}_{60}(\gamma)$ nanoheterostructure interfaces respectively. 


\subsection{Phonon mode analysis, vibrational and optical properties.}

Calculations of vibrational properties of materials from their electronic structures are extremely important goals for materials modeling. Many physical properties of materials depend on their lattice-dynamical behavior; for example, specific heats, thermal expansion, and heat conduction are worth mentioning. Specifically, phenomena related to the electron-phonon interaction, such as the resistivity of metals, superconductivity, and optical spectra's temperature dependence, are just a few of them. Above all, vibrational spectroscopy is a very important tool for the characterization of materials. Vibrational frequencies are consistently and accurately measured using infrared and Raman spectroscopy and inelastic neutron scattering. The resulting vibrational spectra are a sensitive probe of the local bonding and chemical structure. Accurate calculations of frequencies and displacement patterns can thus yield a wealth of information on the atomic and electronic structure of materials. In this section, we first study phonon modes. Next, we investigate the origin of enhancement in polarizability in the $\mathrm{PVK} / \mathrm{C}_{60}$ nanoheterostructure interfaces by studying their vibrational and optical properties. Non-covalent functionalization of PVK- $\mathrm{C}_{60}$ through $\pi-\pi$ and $\pi-\mathrm{H}$ bonds could be a source of charge transfer activity in the nanoheterostructure. The density functional perturbation theory (DFPT) is employed to study the structure, optical phonon modes, and dielectric properties for $\mathrm{PVK}, \mathrm{C}_{60}$, and $\mathrm{PVK} / \mathrm{C}_{60}$ nanoheterostructure interfaces at ambient pressure. To calculate the Born effective charges and high-frequency dielectric tensors accurately, we utilize two sum rules to monitor this calculation.

\subsubsection{Vibrational properties.}

\subsubsection{Phonon dispersions/vibrational analysis.}

Figures 6(a-e) show the first-principles calculated phonon dispersions/vibrational frequency curves for PVK, $\mathrm{C}_{60}, \quad \mathrm{PVK}_{6} / \mathrm{C}_{60}(\alpha), \quad \mathrm{PVK} / \mathrm{C}_{60}(\beta)$, and $\mathrm{PVK} / \mathrm{C}_{60}(\gamma)$ nanoheterostructure interfaces, respectively, along with high-symmetry points of the Brillouin zone (BZ). All the figures show that the phonon dispersions/vibrational curves for the three nanoheterostructure interfaces in the whole Brillouin zone are positive (stable phonon modes) and in good agreement with experimental predictions [34]. It can be seen from Figures 6(c -e) that the highest phonon branches resulting from atomic vibrations are well separated from the other phonon branches. The upper branches of the highest phonon branches are dispersionless or flat mostly along with $\Gamma-\mathrm{N}, \mathrm{Q}-\mathrm{L}$, and $\mathrm{Y}-\mathrm{X}$ directions. In contrast, the lower branches of the lowest phonon branches are dispersive. Far below the gap region, all of the optical branches due to atomic vibrations are dispersive. The calculated phonon dispersions along the highsymmetry lines $\mathrm{L}-\mathrm{Y}-\mathrm{X}$ have been added to illustrate phonon states' whole dispersion in the Brillouin zone (BZ). The low-frequency region branches are the transverse acoustic (TA) and longitudinal acoustic (LA) modes that belong to vibrations of the $\mathrm{C}$ atoms. The other branches are optical modes with higher frequencies associated with lattice vibrations of $\mathrm{N}$ atoms.

In Figures 7(a-e) we present computed phonon density of states (DOS) for (a) PVK, (b) $\mathrm{C}_{60}$, (c) $\mathrm{PVK} / \mathrm{C}_{60}(\alpha)$, (d) $\mathrm{PVK} / \mathrm{C}_{60}(\beta)$, (e) $\mathrm{PVK} / \mathrm{C}_{60}(\gamma)$ nanoheterostructure interfaces respectively. Also, Figures $8(\mathrm{a}-\mathrm{e})$ depicted computed partial phonon density of states (PDOS) for (a) PVK, (b) $\mathrm{C}_{60}$, (c) PVK/C60( $\alpha$ ), (d) PVK/C60( $\beta$ ), (e) PVK/C60 $(\gamma$ ) nanoheterostructure interfaces respectively under investigation. 
(a)

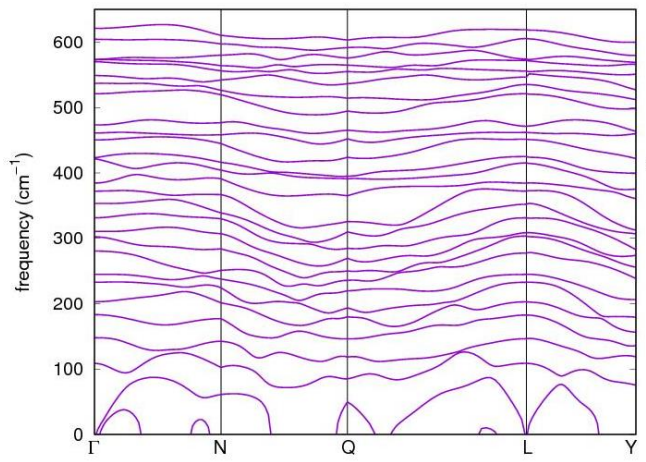

(c)

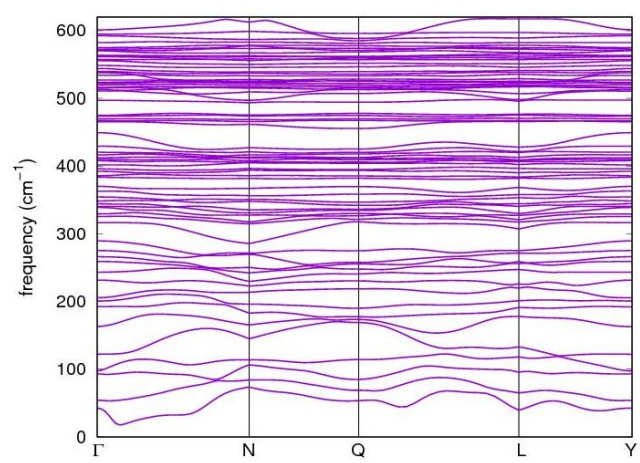

(b)

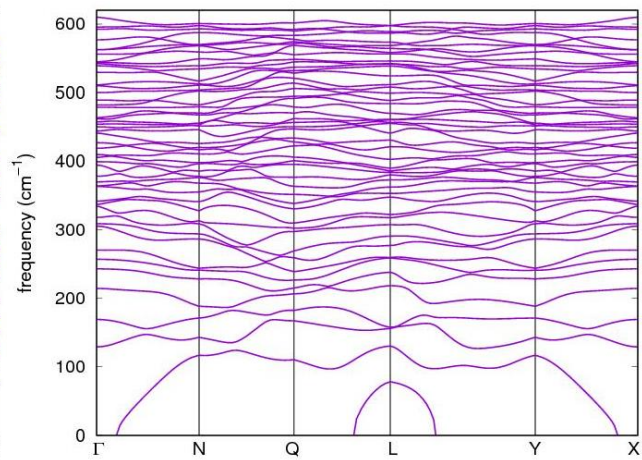

(d)

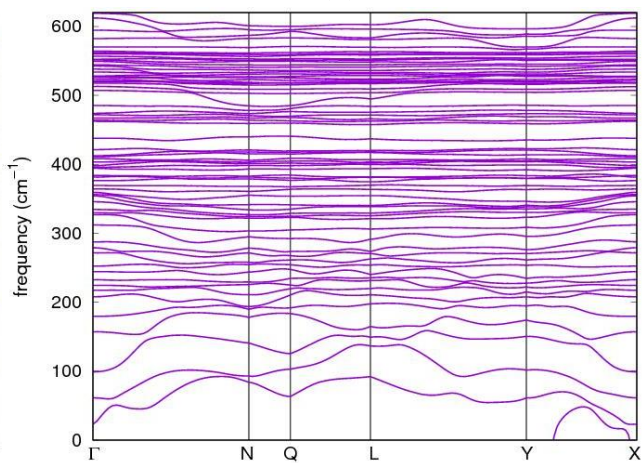

(e)

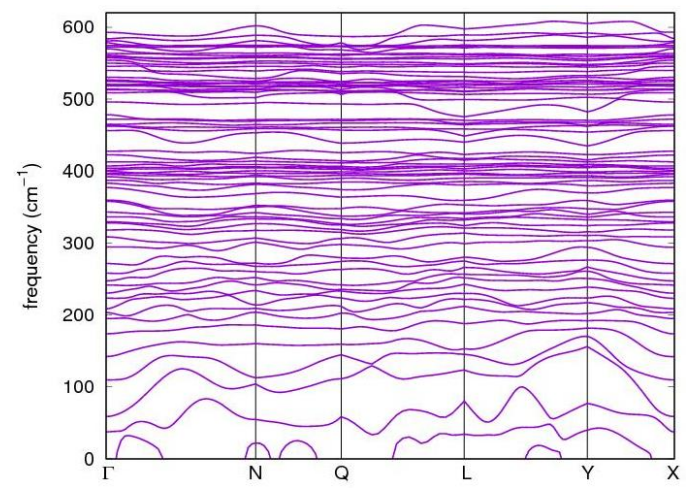

Figure 6. Phonon dispersions/vibrational frequencies for (a) PVK, (b) $\mathrm{C}_{60}$, (c) PVK/C 60 ( $\left.\boldsymbol{\alpha}\right)$, (d) PVK/C 60 ( $\left.\boldsymbol{\beta}\right)$, (e) $\mathrm{PVK} / \mathrm{C}_{60}(\boldsymbol{\gamma})$ nanoheterostructure interfaces respectively.

From Figures 8 (c, d, and e), it can be seen clearly that there are high-intensity peaks at frequencies 860,900 , and $1000 \mathrm{~cm}^{-1}$. Among the several Raman active vibrational modes for carbon atoms, we can attribute these to vibrational modes at radial breathing modes (RBM). At these frequencies, we can infer that the system is vibrating at its maximum peaks. Again, from these figures, it can be seen that the most important (carbon atoms) nanotube modes, the Dand G-bands occur at around $1360 \mathrm{~cm}^{-1}$ and $1600 \mathrm{~cm}^{-1}$, respectively.

It can be seen from Figures 8(c, d, and e) that the PDOS do not extend into the negative range of the spectrum. Suppose the PDOS extends into the negative range. In that case, this may mean that negative frequencies are computed in the irreducible Brillouin zone. In the DOS computation, we need the phonon frequencies in the entire irreducible Brillouin zone. These negative or imaginary phonon frequencies at the Brillouin zone center correspond to acoustic phonon modes or translational vibrations. It is highly probable that these are just numerical noise, and these are pure acoustic modes that should have zero frequencies. But we have numerical noise, which is inevitable with minimization methods used to compute phonons 
since the minimization is never perfect. They may occur from the numerical errors arising during the diagonalization of the dynamical matrix. The results we have obtained from this section have demonstrated that calculations from first principles can be employed in predicting the phonon dispersion modes in agreement with the experiment.

(a)

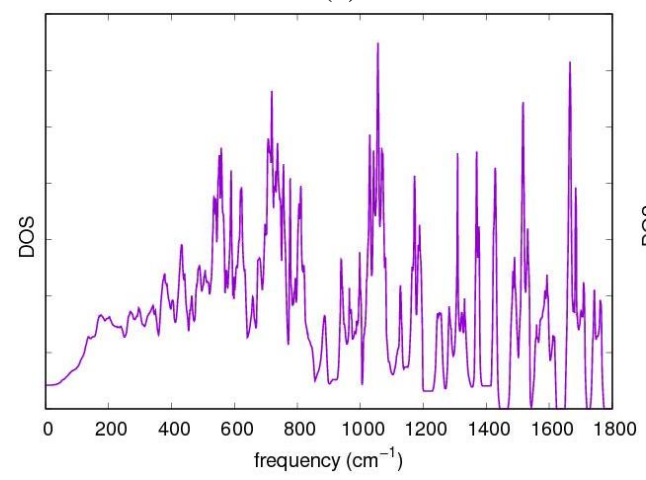

(c)

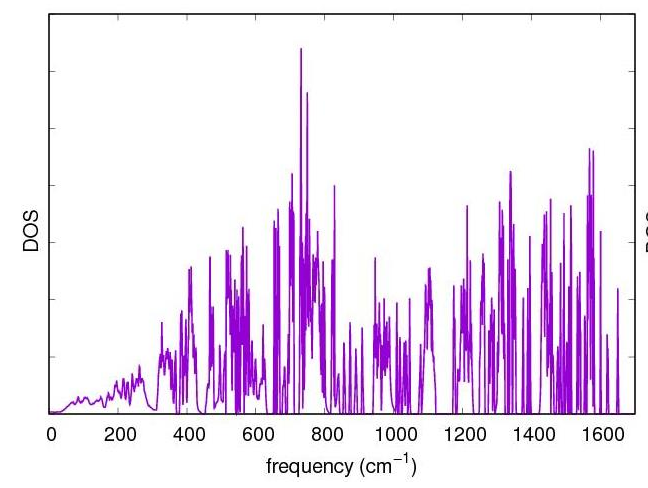

(b)

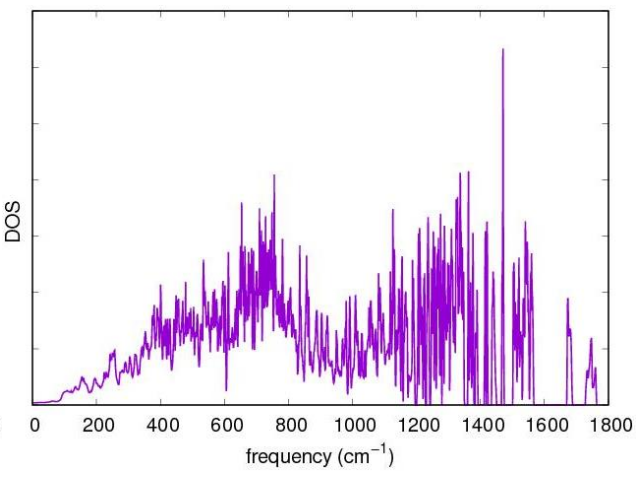

(d)

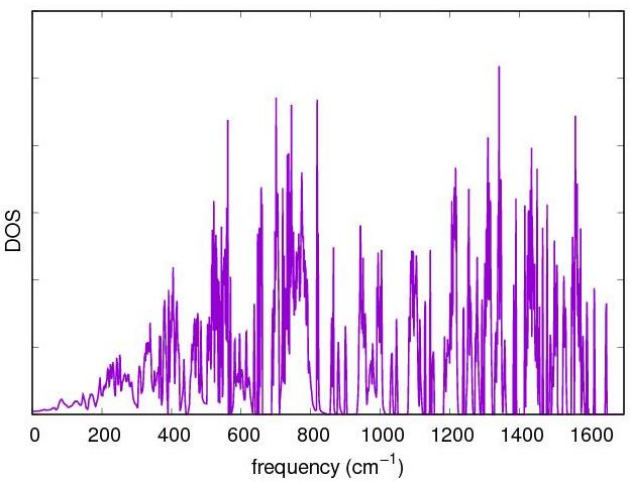

(e)

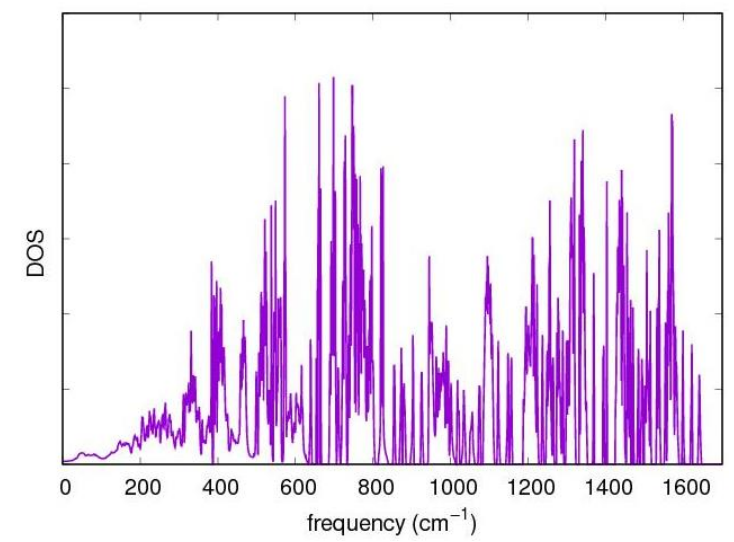

Figure 7. Phonon density of states (DOS) for (a) PVK, (b) $\mathrm{C}_{60}$, (c) PVK/C 60 ( $\alpha$ ), (d) $\mathrm{PVK} / \mathrm{C}_{60}(\beta)$, (e) $\mathrm{PVK} / \mathrm{C}_{60}(\gamma)$ nanoheterostructure interfaces respectively.

3.3.2. Calculated infrared and Raman analysis.

\subsubsection{Infrared analysis.}

Figures $9(\mathrm{a}-\mathrm{e})$ show the vibrational properties, infrared spectra (IR) of (a) PVK, (b) $\mathrm{C}_{60}$, (c) $\mathrm{PVK} / \mathrm{C}_{60}(\alpha)$, (d) $\mathrm{PVK} / \mathrm{C}_{60}(\beta)$, (e) $\mathrm{PVK} / \mathrm{C}_{60}(\gamma)$ nanoheterostructure interfaces respectively. Observations from Figures 9 (c, d, and e) reveal a significant modification in the movement of the PVK vibrational peaks when the $\mathrm{C}_{60}$ is included. As can be seen from Figures 9 (c, d, and e), the calculated IR spectra for PVK without the fullerene, Figure 9(a), and the calculated IR spectra for PVK with the fullerene, Figures 9 (c, d, and e) are different, so this confirms 
interactions involving carbon atoms and charge transfer occurring between PVK matrix and the $\mathrm{C}_{60}$. The infrared spectra of the composite structure reveal bands are appearing at different frequencies in the spectrum.

(a)

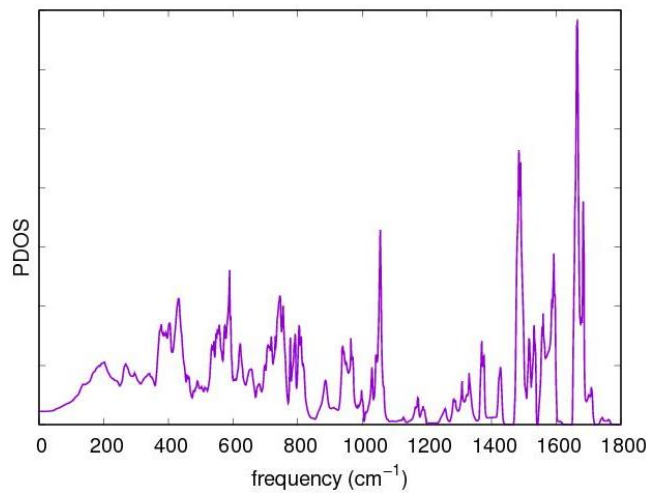

(c)

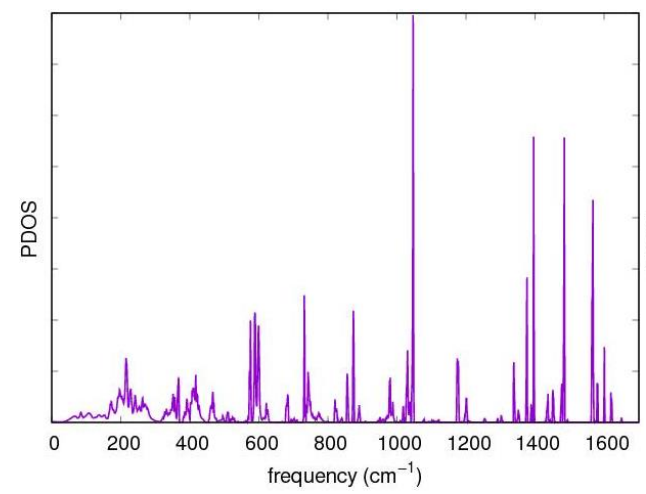

(b)

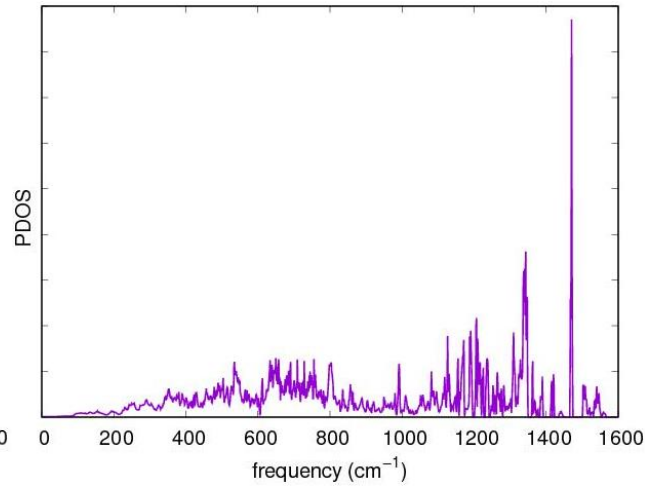

(d)

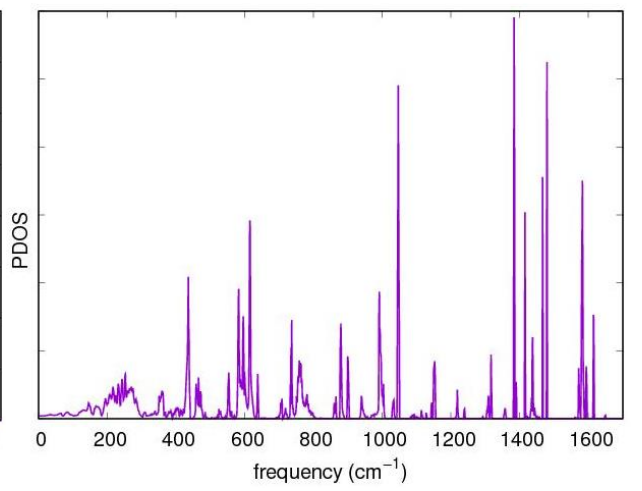

(e)

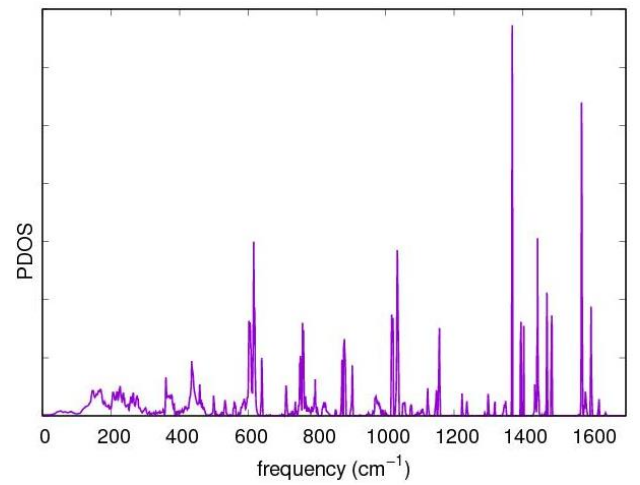

Figure 8. Phonon partial density of states (PDOS) for (a) PVK, (b) $\mathrm{C}_{60}$, (c) PVK/C 60 ( $\left.\boldsymbol{\alpha}\right),(\mathrm{d}) \mathrm{PVK} / \mathrm{C}_{60}(\boldsymbol{\beta})$, (e) $\mathrm{PVK} / \mathrm{C}_{60}(\boldsymbol{\gamma})$ nanoheterostructure interfaces respectively.

It is evident from the calculated infrared spectrum of the $\mathrm{PVK} / \mathrm{C}_{60}$ composite in Figures 9 (c, d, and e) that D- and G-bands appeared at $1350 \mathrm{~cm}^{-1}$ and $1700 \mathrm{~cm}^{-1}$, respectively. The appearance of these bands in the infrared spectra is attributed to $\mathrm{C}-\mathrm{C}$ and $\mathrm{C}=\mathrm{C}$ 's vibrations of carbon atoms in $\mathrm{C}_{60}$ and $\mathrm{PVK}$, which is an indication of non-covalent interaction of $\mathrm{C}_{60}$ with the PVK matrix. The intensity bands observed in Figures 9 (c, d, and e) at 780, 1025, 1220, 1400 , and $1650 \mathrm{~cm}^{-1}$ correspond respectively to the stretching vibrations of $\mathrm{C}=\mathrm{C}$ in the aliphatic chain, $\mathrm{C}-\mathrm{C}$ stretching, $\mathrm{C}-\mathrm{N}$ stretching in $\mathrm{PVK}, \mathrm{CH}_{2}$ deformation of vinylene group, antisymmetric $\mathrm{C}=\mathrm{C}$ stretching of $\mathrm{CH}_{2}$ in $\mathrm{PVK}$ and vibration of $\mathrm{C}=\mathrm{C}$ of carbon nanosphere [34]. 
Table 4. The calculated phonon modes $\left(\right.$ in $\mathrm{cm}^{-1}$ ) and assignments of the three nanoheterostructure interfaces $\mathrm{PVK} / \mathrm{C}_{60}(\alpha), \mathrm{PVK} / \mathrm{C}_{60}(\beta)$ and $\mathrm{PVK} / \mathrm{C}_{60}(\gamma)$ at the $\Gamma$-point in this work.

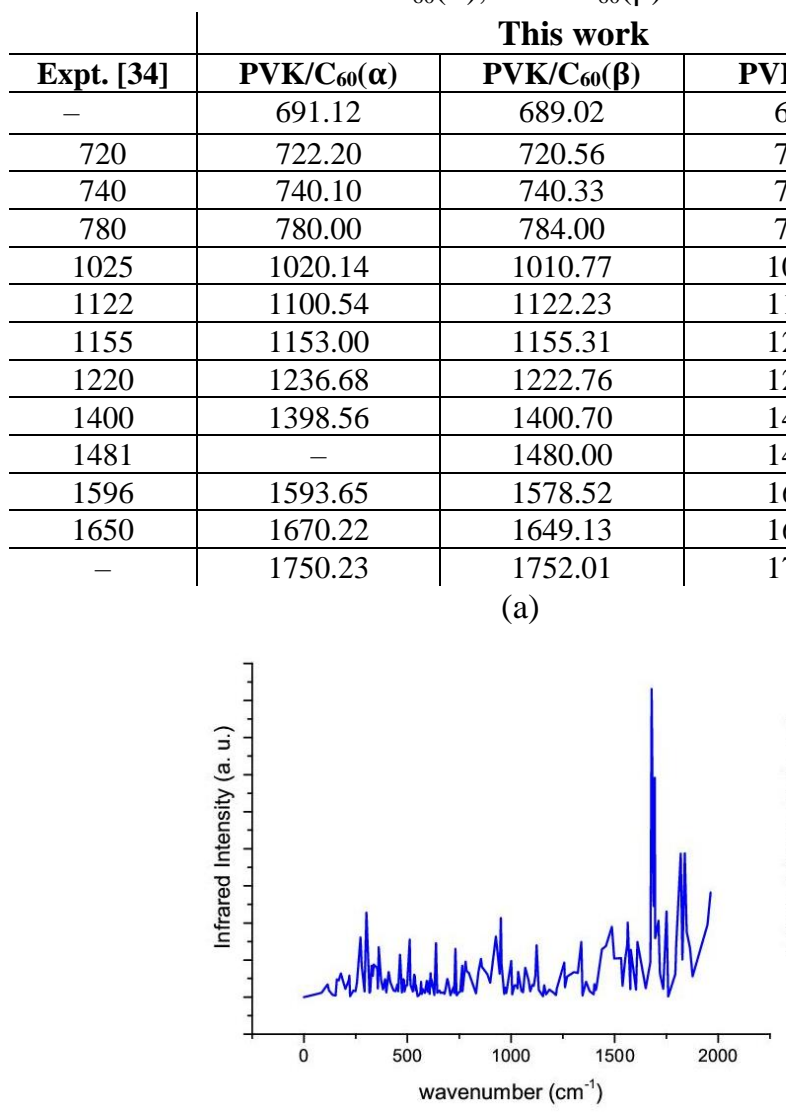

(c)

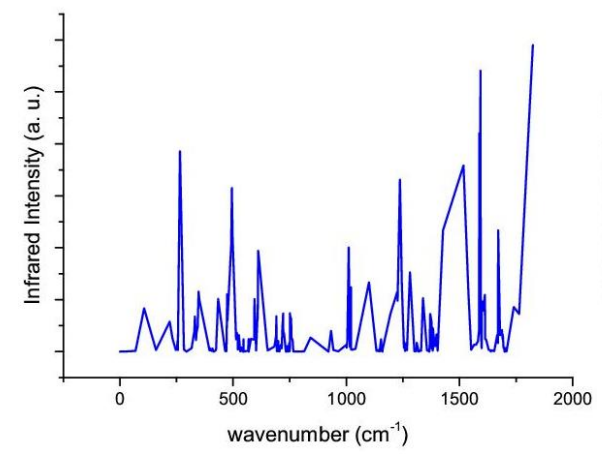

Assignment

\begin{tabular}{|c|c|}
\hline & \\
\hline \multicolumn{2}{|l|}{699.00} \\
\hline 724.00 & aliphatic $\mathrm{C}=\mathrm{C}$ and alkyl groups \\
\hline 745.00 & aliphatic $\mathrm{C}=\mathrm{C}$ and alkyl groups \\
\hline 780.67 & stretching $\mathrm{C}=\mathrm{C}$ aliphatic \\
\hline 1012.38 & stretching C-C \\
\hline 1167.00 & $\mathrm{C}-\mathrm{H}$ of aromatic ring \\
\hline 1235.02 & $\mathrm{C}-\mathrm{H}$ of aromatic ring \\
\hline 1239.58 & C-N stretching of PVK \\
\hline 1435.00 & $\mathrm{CH}_{2}$ deform. of vinyl of PVK \\
\hline 1497.19 & ant. $\mathrm{C}=\mathrm{C}$ stretching of $\mathrm{PVK}$ \\
\hline 1603.31 & $\mathrm{CH}_{2}$ stretching of PVK \\
\hline 1671.14 & vibration of $\mathrm{C}=\mathrm{C}$ of nanosphere \\
\hline
\end{tabular}

(b)

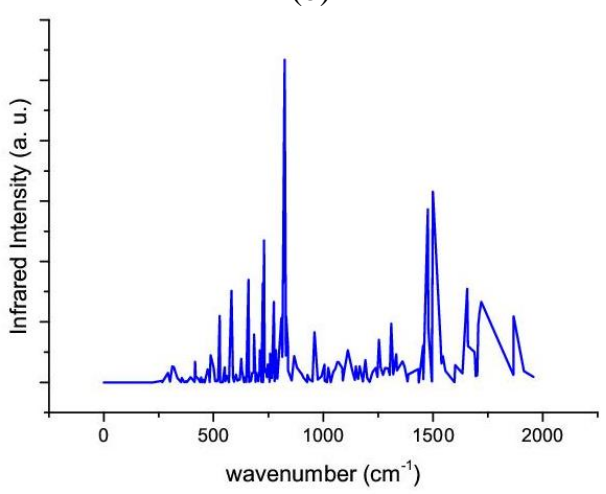

(d)

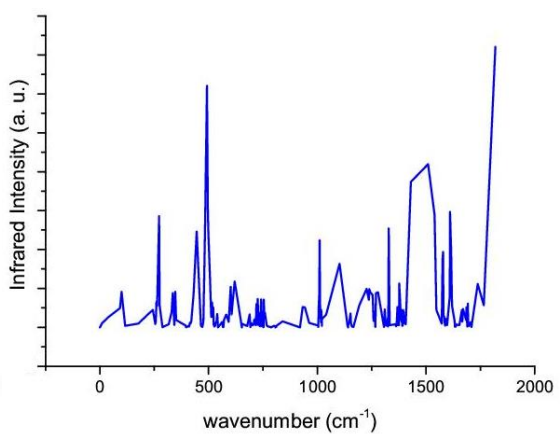

(e)

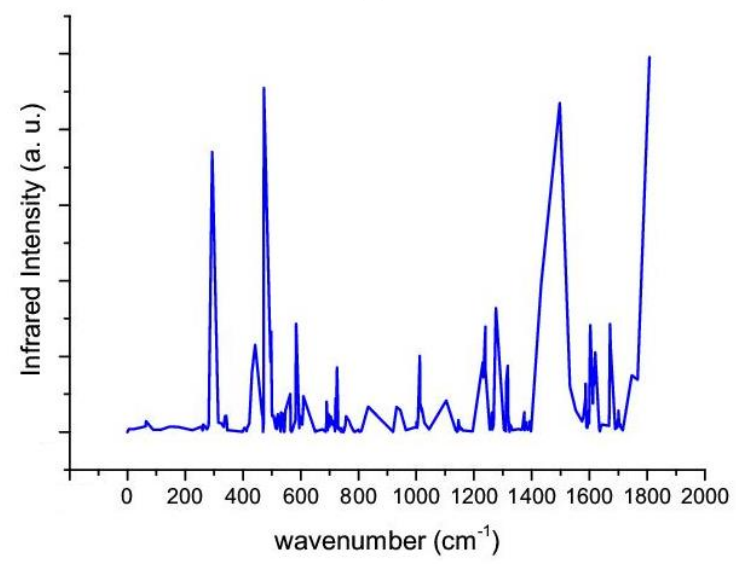

Figure 9. Calculated infrared spectra for (a) PVK, (b) $\mathrm{C}_{60}$, (c) $\mathrm{PVK} / \mathrm{C}_{60}(\alpha)$, (d) $\mathrm{PVK} / \mathrm{C}_{60}(\beta),(\mathrm{e}) \mathrm{PVK} / \mathrm{C}_{60}(\gamma)$ nanoheterostructure interfaces, respectively. 


\subsubsection{Raman analysis.}

The donor character of these functional groups and the presence of $\mathrm{C}_{60}$, which are electron acceptor units, are responsible for the above phenomena. The infrared analysis spectra in this work within the spectral range, $0 \mathrm{~cm}^{-1}$ to $1900 \mathrm{~cm}^{-1}$, thereabout, is in close agreement with experimental results [36,37]. Nonetheless, there are some disagreements between the calculated and experimental results in the range of $2800 \mathrm{~cm}-1$ and above at higher wavenumbers. At these higher frequencies, hydrogen stretching modes, which are very anharmonic, are anticipated. These phenomena normally may be a major cause, so that the calculations may overestimate these by many discrepancies. We speculate that this could also be due to the low polymer chain length used in this work, but this could match with experimental values at higher polymer chain lengths.

(a)

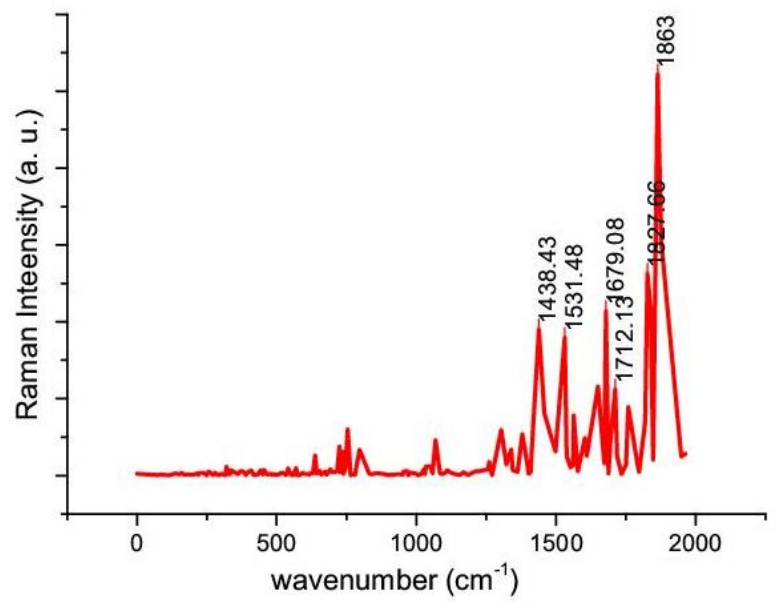

(c)

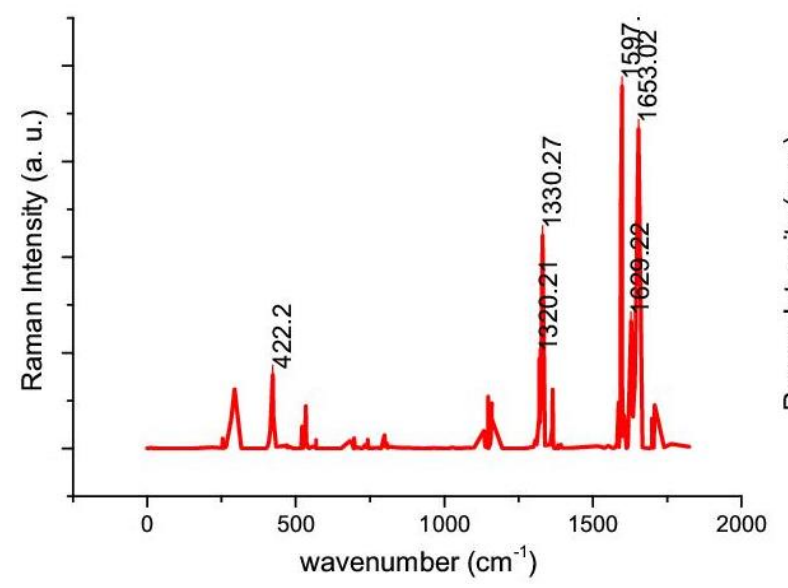

(b)

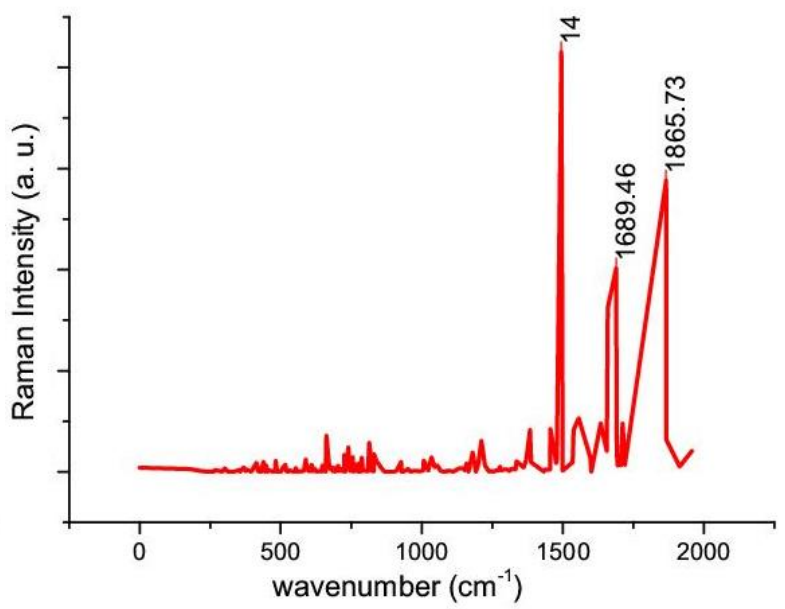

(d)

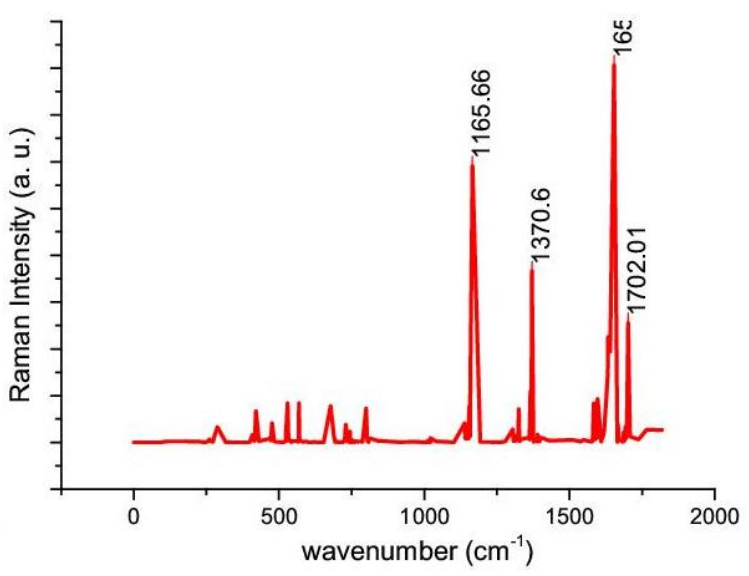

(e)

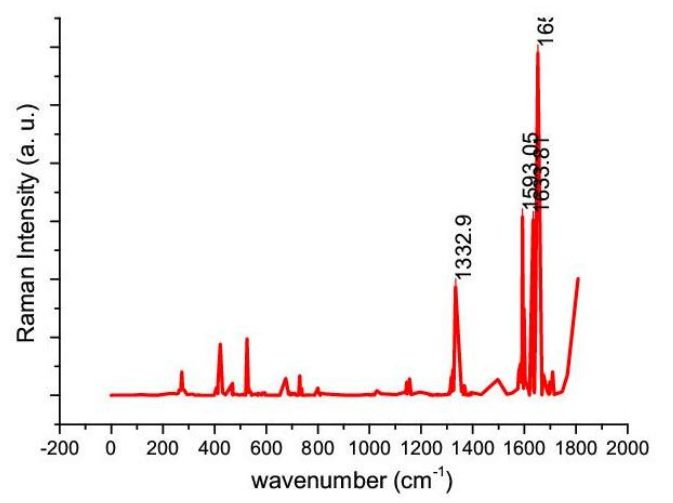

Figure 10. Calculated Raman spectra for (a) $P V K$, (b) $C_{60}$, (c) $P V K / C_{60}(\alpha)$, (d) $P V K / C_{60}(\beta)$, (e) $P V K / C_{60}(\gamma)$ nanoheterostructure interfaces, respectively. 
Table 5. The calculated phonon modes $\left(\right.$ in $\mathrm{cm}^{-1}$ ) and assignments of the three nanoheterostructure interfaces $\mathrm{PVK} / \mathrm{C}_{60}(\alpha), \mathrm{PVK} / \mathrm{C}_{60}(\beta)$ and $\mathrm{PVK} / \mathrm{C}_{60}(\gamma)$ at the $\Gamma$-point in this work.

\begin{tabular}{c|c|c|c|c} 
& \multicolumn{3}{|c}{ This work } & Assignment \\
\hline Expt. [34,35] & PVK/C60 $(\boldsymbol{\alpha})$ & PVK/C60 $(\boldsymbol{\beta})$ & PVK/C60 $(\boldsymbol{\gamma})$ & C-C deformation in polyvinylene \\
\hline- & 291.12 & 251.02 & 299.00 & Benzene ring deformation \\
\hline- & 422.20 & 431.00 & 434.00 & Rocking-Wagg. of methyl in polyvinylene \\
\hline 724 & 722.10 & 724.00 & 725.00 & C-C stretching in PVK \\
\hline 1004 & 1010.00 & 1004.00 & - & Rocking of methyl in polyvinylene \\
\hline 1022 & 1020.00 & 1022.00 & 1022.00 & Rocking-twisting of methylene \\
\hline 1129 & 1130.00 & 1165.66 & 1167.00 & Wagging of methylene in polyvinyl \\
\hline 1235 & 1238.00 & - & 1235.02 & C-H deformation of benzene ring \\
\hline 1320 & 1320.21 & 1370.60 & 1332.90 & C-H deform. in five-mem. ring \\
\hline 1452 & - & 1452.00 & 1435.00 & Stretching of five-member ring \\
\hline 1495 & - & 1495.00 & 1495.00 & C=C stretching in benzene ring \\
\hline 1578 & 1597.00 & 1598.00 & 1593.05 & C-C stretching in benzene ring \\
\hline 1626 & 1620.22 & 1630.00 & 1626.12 & C=C vibration in benzene ring
\end{tabular}

Figures 10 (a - e) show the vibrational properties, Raman spectra for (a) PVK, (b) $\mathrm{C}_{60}$, (c) $\mathrm{PVK} / \mathrm{C}_{60}(\alpha)$, (d) $\mathrm{PVK} / \mathrm{C}_{60}(\beta)$, (e) $\mathrm{PVK} / \mathrm{C}_{60}(\gamma)$ nanoheterostructure interfaces respectively. As seen in Figures $10\left(\mathrm{c}, \mathrm{d}\right.$, and e), the intensity bands located at $724,1022,1320$, and $1626 \mathrm{~cm}^{-1}$ are due to the presence of rocking-wagging of methylene in polyvinylene, rocking of methylene in polyvinylene, $\mathrm{C}$ $\mathrm{H}$ deformation of a benzene ring and $\mathrm{C}-\mathrm{C}$ stretching in benzene rings respectively [34,35]. The calculated Raman intensity is a function of polarizability and symmetry. It authenticates the noncovalent $\pi$-stacking and orientation of $\mathrm{PVK} / \mathrm{C}_{60}$ composite.

\subsubsection{Optical properties}

\subsubsection{Calculated ultraviolet, optical absorption.}

Figures 11(a - e) show calculated optical spectral absorption for (a) PVK, (b) $\mathrm{C}_{60}$, (c) $\mathrm{PVK} / \mathrm{C}_{60}(\alpha)$, (d) $\mathrm{PVK} / \mathrm{C}_{60}(\beta)$, (e) $\mathrm{PVK} / \mathrm{C}_{60}(\gamma)$ nanoheterostructure interfaces respectively. Figures 11(a, b) show the absorption spectra for pristine PVK and $\mathrm{C}_{60}$. Figures $11(\mathrm{c}-\mathrm{e}$ ) were the calculated absorption spectra for the nanoheterostructures when the $\mathrm{C}_{60}$ was added. Figure 11(a) shows that there are five intensity peaks located at $0.41,0.45,0.49,0.51$, and $0.53 \mathrm{Ryd}$. The most intense peak is located at 0.41 Ryd. In Figure 11(b), we observed that there are distinctive absorption levels for each direction. Comparing figure 11(b) with figure 11(c), it is observed that on the addition of the $\mathrm{C}_{60}$, absorption in the $\mathrm{Y}$-direction exceeds absorption in the Z-direction. Also, from figure 11(b), it is observed that absorptions in X-, Y- and Z- directions are almost the same as that of Y-slightly above them. From figure (e), X-direction has the least absorption, with the absorption in the $\mathrm{Y}$ - direction exceeding absorption in the $\mathrm{Z}$ - direction. This observation is an indication of charge transfer processes occurring between the $\mathrm{C}_{60}$ and PVK.

\subsubsection{Electric field response.}

By virtue of perturbation theory, whenever an electric field is applied to a polymer system, the dipole moment's change can always be written as a power series. In that instance, the polarizability tensor can be directly evaluated by finding the second-order derivatives of the total energy, which we obtain in this work using linear response or density functional perturbation theory DFPT $[38,39]$. For practical purposes, it is sufficient to know the first-order changes in the charge density and potential in order to evaluate the change in energy to the second order. It is important to mention here that the electronic charge density's linear response gives the first-order variation in the electronic density induced by the external field. The polarizability $\alpha$ and permittivity $\beta$ for the three nanoheterostructure interfaces have been calculated and discuss in the following sections. 
(a)

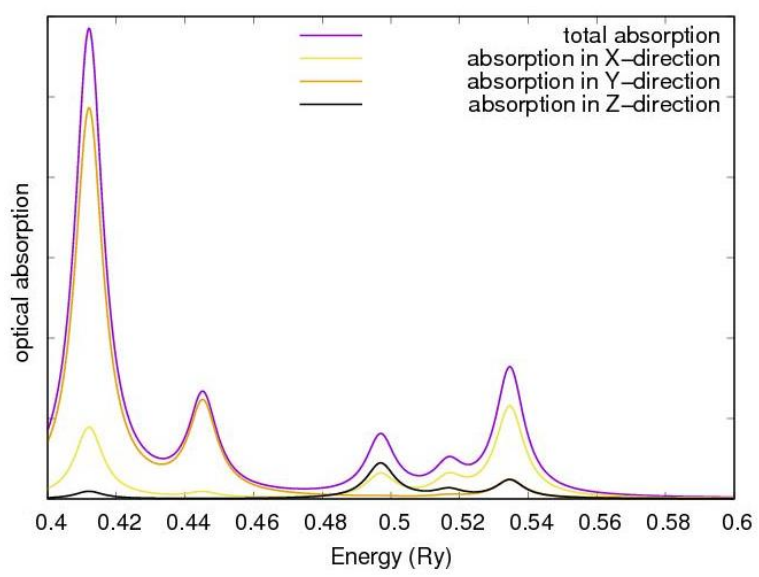

(c)

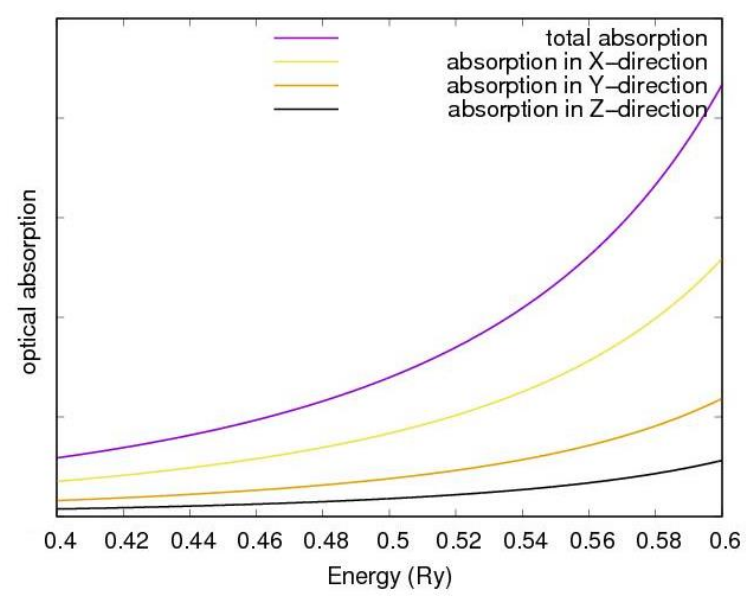

(b)

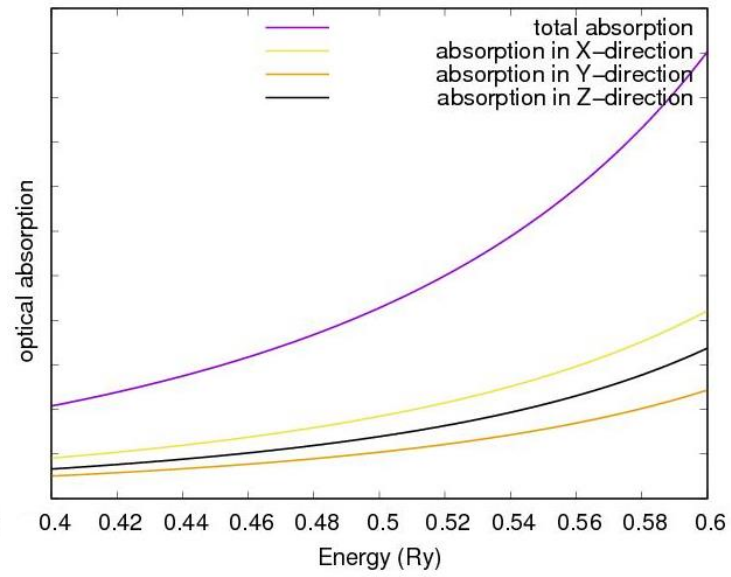

(d)

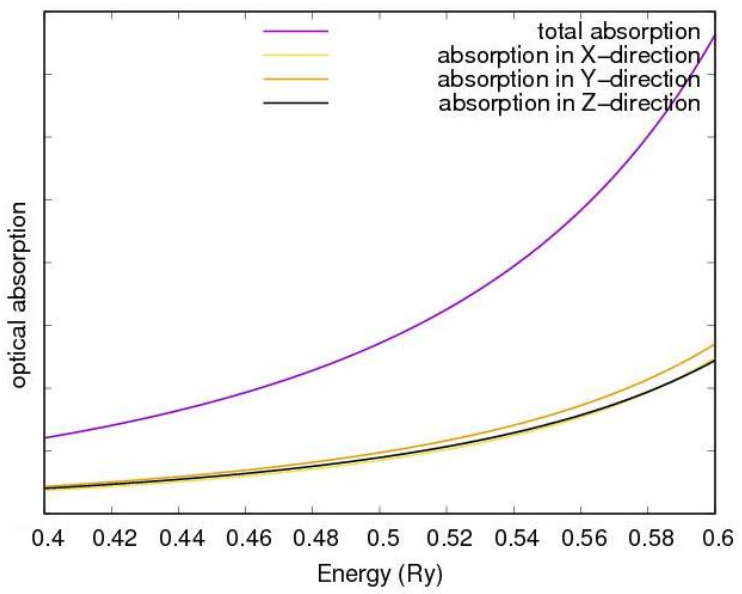

(e)

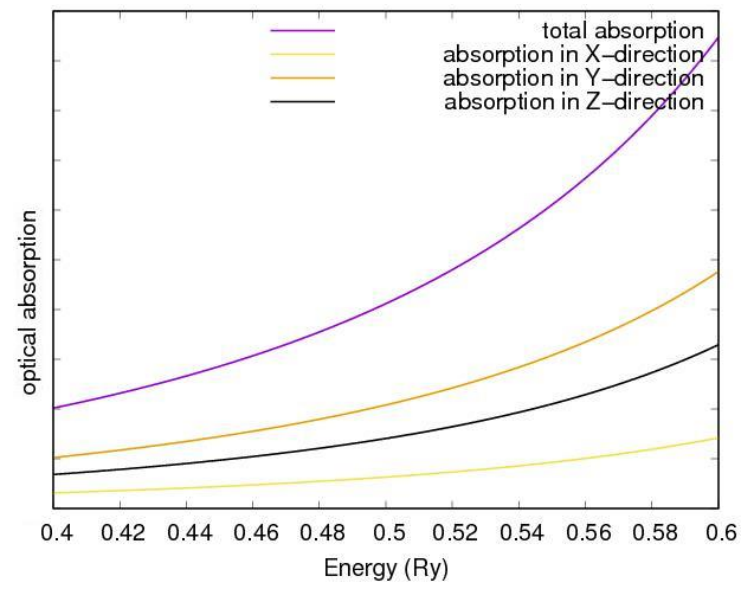

Figure 11. Calculated optical absorption spectra for (a) PVK, (b) $C_{60},(c) P V K / C_{60}(\alpha),(d) P V K / C_{60}(\beta)$, (e) $\mathrm{PVK} / \mathrm{C}_{60}(\gamma)$ nanoheterostructure interfaces respectively.

\subsubsection{Polarizability and permittivity.}

The polarizability and permittivity tensor components calculated using DFPT are given in Table 6. From the Table, it is found that the major contribution to the average polymer polarizability $\alpha_{a v}^{D F P T}$ [ $=\operatorname{Tr}(\alpha) / 3]$ is made by the component $\alpha_{x x}$. For the three nanoheterostructure interfaces, the three individual principal components are very similar to those of an isolated PPV chain [23], which may be attributed to the similarity of the structures of $\mathrm{PVK} / \mathrm{C}_{60}(\alpha), \mathrm{PVK} / \mathrm{C}_{60}(\beta)$, and $\mathrm{PVK} / \mathrm{C}_{60}(\gamma)$. Both $\mathrm{PVK} / \mathrm{C}_{60}(\beta)$ and $\mathrm{PVK} / \mathrm{C}_{60}(\gamma)$ have the property that $\alpha_{x x} \gg \alpha_{y y}$ and $\alpha_{z z} \approx \alpha_{y y}$. For the $\mathrm{PVK} / \mathrm{C}_{60}(\alpha)$, $\alpha_{z z} \gg \alpha_{x x}$ and the component $\alpha_{z z}$ is equal to the component $\alpha_{y y}$ and are larger. From Table 6 , the 
polarizabilities are strongly anisotropic with a relatively large response for an electric field applied along the $y$ and $z$ axes for $P V K / C_{60}(\alpha)$ and $x$-axis for $P V K / C_{60}(\beta)$ and $P V K / C_{60}(\gamma)$.

Table 6. The calculated polarizabilities $\alpha^{D F P T}$ (in $\AA^{3}$ ) and permittivity $\beta$ tensor components for $\operatorname{PVK} / \mathrm{C}_{60}(\alpha)$, $\mathrm{PVK} / \mathrm{C}_{60}(\beta)$ and $\mathrm{PVK} / \mathrm{C}_{60}(\gamma) . \alpha_{a v}^{D F P T}$ and $\beta_{a v}$ are $\operatorname{Tr}(\alpha) / 3$ and $\operatorname{Tr}(\beta) / 3$ respectively.

\begin{tabular}{|c|c|c|c|c|c|c|c|c|}
\hline & \multicolumn{3}{|c|}{$\boldsymbol{\alpha}^{D F P T}$} & \multirow{2}{*}{$\alpha_{a v}^{D F P T}$} & \multicolumn{3}{|c|}{$\boldsymbol{\beta}$} & \multirow{2}{*}{$\boldsymbol{\beta}_{a v}$} \\
\hline \multirow[t]{3}{*}{$\mathrm{PVK} / \mathrm{C}_{60}(\boldsymbol{\alpha})$} & 115.32 & 2.35 & -2.56 & & 3.68 & 0.05 & -0.06 & \\
\hline & 2.35 & 138.87 & 2.69 & & 0.05 & 4.23 & 0.06 & \\
\hline & -2.56 & 2.69 & 138.87 & 131.02 & -0.06 & 0.06 & 4.23 & 4.05 \\
\hline \multirow{3}{*}{$\mathrm{PVK} / \mathrm{C}_{60}(\boldsymbol{\beta})$} & 164.99 & -8.74 & 0.02 & & 5.52 & -0.24 & 0.00 & \\
\hline & $\begin{array}{l}-8.74 \\
\end{array}$ & 118.65 & $\begin{array}{ll}-4.48 \\
\end{array}$ & & -0.24 & 4.25 & -0.12 & \\
\hline & 0.02 & -4.48 & 116.56 & 113.40 & 0.00 & -0.12 & 4.23 & 4.67 \\
\hline \multirow[t]{3}{*}{$\mathrm{PVK} / \mathrm{C}_{60}(\boldsymbol{\gamma})$} & 179.42 & 4.62 & -1.47 & & 6.27 & 0.14 & -0.04 & \\
\hline & $\begin{array}{l}4.62 \\
\end{array}$ & 143.98 & -10.82 & & 0.14 & 5.23 & -0.32 & \\
\hline & -1.47 & -10.82 & 162.40 & 161.93 & -0.04 & -0.32 & 5.77 & 5.76 \\
\hline
\end{tabular}

\subsubsection{Calculated born effective charges.}

Noticeably, $\alpha_{x x}$ for $\mathrm{PVK} / \mathrm{C}_{60}(\alpha)$ is substantially smaller than for $\mathrm{PVK} / \mathrm{C}_{60}(\beta)$ and $\mathrm{PVK} / \mathrm{C}_{60}(\gamma)$. Comparing, it is found that the values of $\alpha_{a v}^{D F P T}$ for the three nanoheterostructures are in comparable agreement with available data for the principal diagonal polarizability of conjugated polymers in Ref. $[40,41]$. The calculated permittivity values of all the nanoheterostructures from Table 6 also show strong anisotropy, with $\beta_{z z} \gg \beta_{x x}$ for $\mathrm{PVK} / \mathrm{C}_{60}(\alpha)$ and $\beta_{z z} \approx \beta_{y y}$ for the rest of them. The average permittivity $\beta_{a v}$ for $\mathrm{PVK} / \mathrm{C}_{60}(\gamma)$ is 5.76 compared to 4.05 for $\mathrm{PVK} / \mathrm{C}_{60}(\alpha)$ and 4.67 for $\mathrm{PVK} / \mathrm{C}_{60}(\beta)$. This phenomenon is comparable to that of PPV for an isolated polymer chain found in Ref. [23]. The calculated values of $\beta_{a v}$ agree reasonably well when compared with the available permittivity values of polymers [37]. By matching the values of the permittivity tensors of the three nanoheterostructures, it is evident that the local field has a greater influence on the permittivity asymmetry of $\mathrm{PVK} / \mathrm{C}_{60}(\gamma)$ than that of $\mathrm{PVK} / \mathrm{C}_{60}(\beta)$. Considering $\mathrm{PVK} / \mathrm{C}_{60}(\beta)$, the principal components of the permittivity tensor show less anisotropy than for $\mathrm{PVK} / \mathrm{C}_{60}(\gamma)$ with $\beta_{x x} \approx \beta_{y y} \approx \beta_{z z}$. This is a manifestation of the fact that the different positions of the nitrogen atom in the various stacking patterns of the $\mathrm{PVK} / \mathrm{C}_{60}$ nanoheterostructure are relevant for the particular structure. In addition, the donor character of the functional groups and the presence of $\mathrm{C}_{60}$, which are electron acceptor units, may also be contributors to the above phenomena.

Table 7 depicts the Born effective charges of each atom in the three nanoheterostructure interfaces. It is well known from the theory that Mulliken population charges are based on the KohnSham orbitals partition and provide information on the static electronic structures. On the contrary, Born effective charges are dependent instead on the system's dynamics and amply furnish us with information concerning the dynamical properties. Consequently, when a dynamic system is the subject of concern, then the concepts of Born effective charges are most appropriate to apply. For this very reason, in this work, we deem it prudent to resort to the latter approach.

We have found from our calculations that all the Born effective charges possess roughly the same trend in value for the hydrogen atoms. The trend manifests that the electronic structure of each hydrogen atom is similar in the three nanoheterostructure polymers. For carbon atoms, except for $\mathrm{C}_{3}$ and $\mathrm{C}_{7}$ in $\mathrm{PVK} / \mathrm{C}_{60}(\beta)$, the Born effective charges are also similar. A careful look at $\mathrm{PVK} / \mathrm{C}_{60}(\alpha)$ reveals there is some positive charge pile-up of 0.047 and 0.086 at the node atom $\mathrm{C}_{2}$ and $0.021,0.232$ for $\mathrm{PVK} / \mathrm{C}_{60}(\beta)$ and $\mathrm{PVK} / \mathrm{C}_{60}(\gamma)$, respectively. The Born effective charges for $\mathrm{C}_{3}$ are 0.120 and for $\mathrm{C}_{4}$ are 0.032, 0.014 and $0.032,0.251$ in $P V K / C_{60}(\alpha), P V K / C_{60}(\beta)$, and $P V K / C_{60}(\gamma)$ respectively. It is most probable that due to the position of the nitrogen atom in the $\mathrm{PVK} / \mathrm{C}_{60}(\alpha)$ stacking pattern, $\mathrm{C}_{7}$ has a much less negative charge of -0.001 compare to its contemporaries $\mathrm{C}_{7}$ in $\mathrm{PVK} / \mathrm{C}_{60}(\beta)$ and $\mathrm{PVK} / \mathrm{C}_{60}(\gamma)$. Marked differences exist between the average Born effective charges, $Z_{a v}^{*}$, of the nitrogen atoms -0.918 , -1.005, and -1.358 for $\mathrm{PVK} / \mathrm{C}_{60}(\alpha), \mathrm{PVK} / \mathrm{C}_{60}(\beta)$, and $\mathrm{PVK} / \mathrm{C}_{60}(\gamma)$ respectively, based on the respective stacking orientation. 
Table 7. The calculated Born effective charges $Z_{\alpha \beta}^{*}$ for the three nanoheterostructure interfaces $\mathrm{PVK} / \mathrm{C}_{60}(\alpha)$, $\mathrm{PVK} / \mathrm{C}_{60}(\beta)$ and $\mathrm{PVK} / \mathrm{C}_{60}(\gamma) . Z_{a v}^{*}$ is the average of the principal components. All the values are in units of e.

\begin{tabular}{|c|c|c|c|c|c|c|c|c|c|c|c|c|}
\hline & \multicolumn{4}{|c|}{$\mathrm{PVK} / \mathrm{C}_{60}(\boldsymbol{\alpha})$} & \multicolumn{4}{|c|}{$\mathrm{PVK} / \mathrm{C}_{60}(\boldsymbol{\beta})$} & \multicolumn{4}{|c|}{$\mathrm{PVK} / \mathrm{C}_{60}(\boldsymbol{\gamma})$} \\
\hline & $Z_{x x}^{*}$ & $Z_{y y}^{*}$ & $Z_{z Z}^{*}$ & $Z_{a v}^{*}$ & $Z_{x x}^{*}$ & $Z_{y y}^{*}$ & $\boldsymbol{Z}_{\mathbf{Z Z}}^{*}$ & $Z_{a v}^{*}$ & $Z_{x x}^{*}$ & $Z_{y y}^{*}$ & $Z_{z Z}^{*}$ & $Z_{a v}^{*}$ \\
\hline $\mathrm{N}_{1}$ & -0.351 & -1.165 & -1.238 & -0.918 & -1.306 & -0.711 & -0.999 & -1.005 & -2.147 & -0.942 & -0.984 & -1.358 \\
\hline $\mathrm{C}_{2}$ & -0.442 & 0.047 & 0.086 & -0.103 & 0.021 & -0.252 & -0.093 & -0.108 & 0.232 & -0.373 & -0.110 & -0.084 \\
\hline $\mathrm{C}_{3}$ & -0.442 & 0.120 & -0.290 & -0.204 & -0.373 & -0.277 & -0.106 & 0.252 & -0.137 & -0.372 & -0.388 & -0.299 \\
\hline $\mathrm{C}_{4}$ & -0.328 & -0.009 & 0.032 & -0.102 & 0.014 & -0.283 & -0.077 & -0.115 & 0.032 & -0.333 & 0.251 & -0.017 \\
\hline $\mathrm{C}_{5}$ & -0.375 & -0.156 & 0.086 & -0.148 & 0.162 & -0.337 & -0.174 & -0.116 & -0.260 & -0.063 & 0.171 & -0.051 \\
\hline $\mathrm{C}_{6}$ & -0.152 & 0.948 & -0.281 & 0.172 & -0.076 & 0.393 & 0.674 & 0.330 & 0.151 & 0.197 & 0.730 & 0.359 \\
\hline $\mathrm{C}_{7}$ & -0.400 & -0.263 & -0.001 & -0.221 & -0.114 & -0.450 & -0.377 & 0.314 & -0.204 & -0.273 & -0.064 & -0.180 \\
\hline $\mathrm{H}_{8}$ & 0.236 & 0.101 & 0.007 & 0.115 & -0.005 & 0.263 & 0.141 & 0.133 & 0.085 & 0.369 & 0.085 & 0.180 \\
\hline $\mathrm{H}_{9}$ & 0.216 & 0.027 & 0.030 & 0.091 & 0.115 & 0.214 & 0.068 & 0.132 & 0.089 & 0.302 & -0.036 & 0.118 \\
\hline $\mathrm{H}_{10}$ & 0.266 & 0.105 & 0.000 & 0.124 & -0.007 & 0.373 & 0.092 & 0.153 & -0.062 & 0.372 & 0.143 & 0.151 \\
\hline $\mathrm{H}_{11}$ & 0.262 & 0.032 & 0.266 & 0.176 & 0.196 & 0.158 & 0.174 & 0.176 & -0.036 & 0.397 & 0.205 & 0.189 \\
\hline $\mathrm{H}_{12}$ & 0.259 & -0.019 & 0.144 & 0.128 & 0.123 & 0.227 & 0.056 & 0.135 & 0.089 & 0.254 & 0.079 & 0.141 \\
\hline $\mathrm{H}_{13}$ & 0.201 & 0.038 & 0.065 & 0.101 & 0.103 & 0.172 & 0.128 & 0.134 & 0.028 & 0.253 & 0.131 & 0.137 \\
\hline
\end{tabular}

To gain an insight into the reasons for the discrepancies, we deem it prudent to make a one-onone comparison among the average Born significant charges. Considering the hydrogen atoms, there are apparent differences observed among the average Born significant charges. Among the three nanoheterostructures, the Born significant charges look alike with the exception of $\mathrm{H}_{9}$ with 0.091 , about one-half of the Born significant charges of its correspondents in $\mathrm{PVK} \mathrm{C}_{60}(\beta)$ and $\mathrm{PVK} / \mathrm{C}_{60}(\gamma)$. In the case of the carbon atoms, there exist substantial varieties among the average Born significant charges. Taking $\mathrm{PVK} / \mathrm{C}_{60}(\gamma)$ into consideration, the significant discrepancy is seen in $\mathrm{C}_{4}$ with 0.017 , but of course, values of all $Z_{a v}^{*}$ do have the same sign. On the contrary, there are differences in charge signs for atoms $\mathrm{C}_{6}$ for all three nanoheterostructures. The nitrogen atom's position with its electron donoracceptor characteristics in the ring may be responsible for this occurrence. The Born effective charge is a quantity that relates the dynamical properties of a system directly, more importantly, to the electronic structure changes. As such, effects on the chemical environment considering neighboring chemical bonds are likely to have an adverse effect on the charges. Inspection of Table 7 reveals that the nitrogen atom has a smaller negative effective charge of -0.918 in $\mathrm{PVK} / \mathrm{C}_{60}(\alpha)$ than that of -1.005 and -1.358 , respectively, in $\mathrm{PVK} / \mathrm{C}_{60}(\beta)$ and $\mathrm{PVK} / \mathrm{C}_{60}(\gamma)$. We attribute this to the different bonding effects in the nanoheterostructures depending on stacking patterns. The various variations described among the Born effective charges for the three nanoheterostructure interfaces highlight their different chemical environmental effects based on stacking patterns.

\section{Conclusions}

Employing ab initio density functional methods, we explore the dynamical properties of the three nanoheterostructure interfaces $\mathrm{PVK} \mathrm{C}_{60}(\alpha), \mathrm{PVK}_{6} / \mathrm{C}_{60}(\beta)$, and $\mathrm{PVK} / \mathrm{C}_{60}(\gamma)$ in this work. Using linear response determination of the second-order change in the total energy induced by atomic displacements, we compute the vibrational frequencies of the nanoheterostructures by directly evaluating the dynamical matrix of the force constants. The computed vibrational frequencies are assigned to the phonon modes exhibited by the nanoheterostructure interfaces. Classifications of the phonon modes revealed that the higher frequency modes are due to $\mathrm{C}-\mathrm{C}$ stretching in the benzene ring, the vibration of $\mathrm{C}=\mathrm{C}$ of Nanosphere, $\mathrm{CH}_{2}$ stretching of PVK, and C-N stretching of PVK. Our calculated polarizability and permittivity tensors for the nanoheterostructures in the current work agree well with experimental values for conjugated polymers. The dynamical Born significant charges calculated in this work have been reported and are comparable with experimental values. Effective charges are more appropriate in studying the dynamical properties of our systems 
since they are dynamic systems. Peculiar differences are noticed in the computed dynamical properties of the nanoheterostructures $\mathrm{PVK} / \mathrm{C}_{60}(\alpha), \mathrm{PVK} / \mathrm{C}_{60}(\beta)$, and $\mathrm{PVK} / \mathrm{C}_{60}(\gamma)$ studied. These dynamical properties are relevant in enhancing the transport properties of the nanoheterostructure interfaces. The permittivity and polarizability tensors of $\mathrm{PVK} / \mathrm{C}_{60}(\alpha)$, $\mathrm{PVK} / \mathrm{C}_{60}(\beta)$, and $\mathrm{PVK} / \mathrm{C}_{60}(\gamma)$ nanoheterostructures are found to be strongly anisotropic.

\section{Funding}

This research received no external funding.

\section{Acknowledgments}

A.Y. acknowledges financial support from BANGA-Africa, University of Ghana, and the Carnegie cooperation of New York, USA, for travel grants. V.W.E. acknowledges financial support from the University of Ghana Pan African Doctoral Academy (PADA). Authors are grateful to the Centre for High-Performance Computing (CHPC), Cape Town, South Africa, for computer time on the Lengau cluster.

\section{Conflicts of Interest}

The authors declare no conflict of interest.

\section{References}

1. Wang, R.; Lin, K.; Jiang, F.; Zhou, W.; Wang, Z.; Wu, Y.; Ding, Y.; Hou, J.; Nie, G.; Xu, J.; Duan, X. Fluoro-substituted conjugated polyindole for desirable electrochemical charge storage materials. Electrochimica Acta 2019, 320, https://doi.org/10.1016/j.electacta.2019.134641.

2. Elloh, V.W.; Abhishek Mishra, K.; Dodoo-Arhin, D.; Eric Abavare, K.K.; Gebreyesus, G.; Nyankson, E.; Efavi, J.K.; Onwona-Agyeman, B.; Yaya, A. Structural and Electronic properties of PVK/C60 Nanoheterostructure interfaces - A DFT Approach. Journal of Surfaces and Interfaces 2020, 20 , https://doi.org/10.1016/j.surfin.2020.100556.

3. Rehman, A.; Park, S.J. Comparative study of activation methods to design nitrogen-doped ultra-microporous carbons as efficient contenders for $\mathrm{CO}_{2}$ capture. Chem. Eng. J. 2018, 352, 539-548, https://doi.org/10.1016/j.cej.2018.07.046.

4. Rehman, A.; Park, S.J. Tunable nitrogen-doped microporous carbons: delineating the role of optimum pore size for enhanced $\mathrm{CO}_{2}$ adsorption. Chem. Eng. J. 2019, 362, 731-742, https://doi.org/10.1016/j.cej.2019.01.063.

5. Yue, L.; Xia, Q.L.; Wang, L.; Wang, H.; Da Costa, J.; Yang, X.; Hu. $\mathrm{CO}_{2}$ adsorption at nitrogen-doped carbons prepared by $\mathrm{K}_{2} \mathrm{CO}_{3}$ activation of urea-modified coconut shell. J. Colloid Interface Sci. 2018, 511, 259-267, https://doi.org/10.1016/j.jcis.2017.09.040.

6. Kim, D.K.; Bong, S.X.; Jin, K.; Seong, M.; Hwang, N.D.; Kim, N.; You, Y.; Piao. Facile in situ synthesis of multiple-heteroatom-doped carbons derived from polyimide precursors for flexible all-solid-state super capacitors. ACS Appl. Mater. Interfaces 2018, 11, 1996-2005, https://doi.org/10.1021/acsami.8b15162.

7. Elloh, V.W.; Apalangya, V.A.; Gebreyesus, G.; Abhishek, K.M.; Kan-Dapaah, K.; Nyankson, E.; Efavi, J.K.; Onwona-Agyeman, B.; Yaya, A. A theoretical study of the structural and electronic properties of poly (9-vinylcarbazole) interacting with small diameter single-walled carbon nanotubes. Inte. Jour. of Comput. Mater. Sci. \& Engi. 2020, https://doi.org/10.1142/S2047684120500098.

8. Mateos, M.; Meunier-Prest, R.; Heintz, O.; Herbst, F.; Suisse, J.M.; Bouvet, M. Comprehensive study of poly (2,3,5,6-tetrafluoroaniline): from electro synthesis to heterojunctions and ammonia sensing. ACS Appl. Mater. Interfaces 2018, 10, 19974-19986, https://doi.org/10.1021/acsami.8b03601.

9. Kayang, K.W.; Nyankson, E.; Efavi, J.K.; Abavare, E.K.K.; Garu, G.; Onwona-Agyeman, B.; Yaya, A. Single-Walled boron nitride nanotubes interaction with nickel, titanium, palladium, and gold metal atomsA first-principles study. Results in Materials 2019, 2, https://doi.org/10.1016/j.rinma.2019.100029.

10. Heo, Y.J.; Lee, J.W.; Son, Y.R.; Lee, J.H.; Yeo, C.S.; Lam, T.D.; Park, S.Y.; Park, S.J.; Sinh, L.H.; Shin, M.K. Large-scale conductive yarns based on twistable. Korean Traditional Paper (Hanji) for super capacitor applications: toward high-performance paper super capacitors. Adv. Energy Mater. 2018, 8, https://doi.org/10.1002/aenm.201801854. 
11. Elloh, Van W.; Abu Yaya; Gebreyesus, G.; Piyush Dua; Mishra, Abhishek K. New 2D Structural Materials: Carbon-Gallium Nitride (CC-GaN) and Boron-Gallium Nitride (BN-GaN) Heterostructures - Materials Design Through Density Functional Theory. Journal of American Chemical Society (ACS) Omega 2019, 4, 1722-1728, https://doi.org/10.1021/acsomega.8b03025.

12. Yaya, A.; Impellizzeri, A.; Massuyeau, F.; Duvail, J.L.; Patrick Briddon; Ewels, C.P. Mapping the stacking interaction of Triphenyl vinylene oligomers with graphene and carbon nanotubes. Carbon 2019, 141, 274282, https://doi.org/10.1016/j.carbon.2018.09.071.

13. Wang, L.; Rao, L.; Xia, B.; Wang, L.; Yue, L.; Liang, Y.; Da-Costa, H.; Hu, X. Highly efficient $\mathrm{CO}_{2}$ adsorption by nitrogen-doped porous carbons synthesized with low temperature sodium amide activation. Carbon 2018, 130, 31-40, https://doi.org/10.1016/j.carbon.2018.01.003.

14. Kayang, K.W.; Nyankson, E.; Efavi, J.K.; Apalangya, V.A.; Adetunji, B.I.; Gebreyesus, G.; Tia, R.; Abavare, E.K.K.; Onwona-Agyeman, B.; Yaya, A. A comparative study of the interaction of nickel, titanium, palladium, and gold metals with single-walled carbon nanotubes: A DFT approach. Results in Physics 2019, 12, 2100-2106, https://doi.org/10.1016/j.rinp.2019.02.062.

15. Singh, G.; Lakhi, K.S.; Ramadass, K.; Kim, S.; Stockdale, D.; Vinu, A. A combined strategy of acid-assisted polymerization and solid state activation to synthesize functionalized nanoporous activated biocarbons from biomass for $\mathrm{CO}_{2}$ capture. Microporous Mesoporous Mater. 2018, 271, 23-32, https://doi.org/10.1016/j.micromeso.2018.05.035.

16. Luo, L.; Chen, T.; Li, Z.; Zhang, Z.; Zhao, W.; Fan, M. Heteroatom self-doped activated biocarbons from fir bark and their excellent performance for carbon dioxide adsorption. J. $\mathrm{CO}_{2}$ Util. 2018, 25, 89-98, https://doi.org/10.1016/j.jcou.2018.03.014.

17. Yu, D.; Hu, J.; Zhou, L.; Li, J.; Tang, J.; Peng, C.; Liu, H. Nitrogen-doped coal tar pitch based microporous carbons with superior $\mathrm{CO}_{2}$ capture performance. Energy Fuels 2018, 32, 3726-3732, https://doi.org/10.1021/acs.energyfuels.8b00125.

18. Singh, M.G.; Lakhi, K.S.; Park, D.H.; Srivastava, P.; Naidu, Vinu, R.A. Facile one-pot synthesis of activated porous biocarbons with a high nitrogen content for $\mathrm{CO}_{2}$ capture. Chem. Nano Mat. 2018, 4 281-290, https://doi.org/10.1002/cnma.201700348.

19. Elloh, V.W.; Mishra, S.; Yaya, A.; Mishra, A.K. First-principles calculations on structure and electronic properties of $\alpha$-zirconium hydrogen phosphate. Journal of MRS Advances, Materials Research Society 2019, 4, 2699-2707, https://doi.org/10.1557/adv.2019.309.

20. Rehman, A.; Park, S.J. Comparative study of activation methods to design nitrogen doped ultra-microporous carbons as efficient contenders for $\mathrm{CO}_{2}$ capture. Chem. Eng. J. 2018, 352, 539-548, https://doi.org/10.1016/j.cej.2018.07.046.

21. Tian, Z.; Huang, J.; Zhang, X.; Shao, G.; He, Q.; Cao, S.; Yuan, S. Ultra-microporous N-doped carbon from poly-condensed framework precursor for $\mathrm{CO}_{2}$ adsorption. Micropor. Mesopor. Mater. 2018, 257, 19-26, https://doi.org/10.1016/j.micromeso.2017.08.012.

22. Bera, R.; Mondal, S.; Das, N. Triptycene based microporous polymers (TMPs): efficient small gas $\left(\mathrm{H}_{2}\right.$ and $\mathrm{CO}_{2}$ ) storage and high $\mathrm{CO}_{2} / \mathrm{N}_{2}$ selectivity. Micropor. Mesopor. Mater. 2018, 257, 253-261, https://doi.org/10.3389/fenrg.2019.00141.

23. Elloh, V.; Yaya, A.; Mishra, A.K. Density Functional Theory (DFT) Study of Structural and Electronic Properties of Crystalline 2,3-benzanthracene. International Journal of Engineering, Science and Mathematics 2018, 7, 4-8.

24. Giannozzi, P.; Andreussi, O.; Brumme, T.; Bunau, O.; Buongiorno Nardelli, M.; Calandra, M.; Car, R.; Cavazzoni, C.; Ceresoli, D.; Cococcioni, M.; Colonna, N.; Carnimeo, I.; Dal Corso, A.; De Gironcoli, S.; Delugas, P.; Distasio, R. A.; Ferretti, A.; Floris, A.; Fratesi, G.; Fugallo, G.; Gebauer, R.; Gerstmann, U.; Giustino, F.; Gorni, T.; Jia, J.; Kawamura, M.; Ko, H.Y.; Kokalj, A.; Kucukbenli, E.; Lazzeri, M.; Marsili, M.; Marzari, N.; Mauri, F.; Nguyen, N. L.; Nguyen, H.V.; Otero-De-La-Roza, A.; Paulatto, L.; Ponce, S.; Rocca, D.; Sabatini, R.; Santra, B.; Schlipf, M.; Seitsonen, A.P.; Smogunov, A.; Timrov, I.; Thonhauser, T.; Umari, P.; Vast, N.; Wu, X.; Baroni, S. Advanced capabilities for materials modelling with Quantum ESPRESSO. J. Phys. Condens. Matter. 2017, 29..

25. http://www.quantum-espresso.org.

26. Rappe, A.M.K.; Rabe, M.; Kaxiras, E.; Joannopoulos, J.D. Optimized pseudopotentials Phys. Rev. 1990, 41, 1227-1230, https://doi.org/10.1103/PhysRevB.41.1227.

27. Perdew, J.; Burke, K.; Ernzerhof, M. Generalized gradient approximation made simple. Phys. Rev. Lett. 1996, 77, 3865-3868, https://doi.org/10.1103/PhysRevLett.77.3865.

28. Torbjorn, B.; Granas, O. Adaptive Smearing for Brillouin Zone Integration. Int. Jour. of Quan. Chem. 2011, 111, https://doi.org/10.1002/qua.22476.

29. Fletcher, R. Practical Methods of Optimization. New York, Wiley 1987.

30. Monkhorst, H.J.; Pack, J.D. Special points for Brillouin-zone integrations. Physical Review B 1976, 13, 5188-5192, https://doi.org/10.1103/PhysRevB.13.5188.

31. Marcus, R.A. Electron transfer reactions in chemistry. Theory and experiment. Reviews of Modern Physics 1993, 65, 599-610, https://doi.org/10.1103/RevModPhys.65.599. 
32. Ramon, J.G.S.; Bittner, E.R. Exciton Regeneration Dynamics in Model Donor-Acceptor Polymer Heterojunctions. The Journal of Physical Chemistry B 2006, 110, 21001-21009, https://doi.org/10.1021/jp061751b.

33. Hu, X.; Zhang, Y.; Zhuang, N.; Li, J. Density functional theory predictions for blue luminescence and nonlinear optical properties of carbon-doped gallium nitride. Journal of Solid State Chemistry 2010, 183, 2741-2745, https://doi.org/10.1016/j.jssc.2010.08.010.

34. Baibarac, M.; Baltog, I.S.; Lefrant, P.; Gomez-Romero. Spectroscopic evidence for the bulk polymerization of N-vinyl carbazole in the presence of single-walled carbon nanotubes. Polymer 2007, 48, 5279-5288, https://doi.org/10.1016/j.polymer.2007.07.008.

35. Journet, C.; Maser, W.K.; Bernier, P.; Loiseau, A.; de la Chapelle, M.L.; Lefrant, S.; Deniard, P.; Lee, R.; Fischer, J.E. Large-scale production of single-walled carbon nanotubes by the electric-arc technique. Nature 1997, 388, 756-758, https://doi.org/10.1038/41972.

36. Mbarek, M.; Ghnimi, M.F.; Alimi Abbassi, K. Novel carbon nanospheres and poly(9vinylcarbazole) composites, synthesis, structural and photo-physical properties of films elaboration. Journal of Materials Chemistry and Physics 2018, 211 399-405, https://doi.org/10.1016/j.matchemphys.2018.03.006.

37. Chemek, M.; Khlaifia, D.; Massuyeau, F.; Duvail, J. L.; Faulques, E.; Wéry, J.; Alimi, K. A Copolymer of PVK and P3HT and its nanocomposite with single-walled carbon nanotubes. Synthetic Metals 2014, 197, 246-251, https://doi.org/10.1016/j.synthmet.2014.09.004.

38. Gonze, X. Dynamical matrices, Born effective charges, dielectric permittivity tensors, and interatomic force constants from density-functional perturbation theory. Phys. Rev. 1996, B 55, https://doi.org/10.1103/PhysRevB.55.10355.

39. Baroni, S.; de Gironcoli, S.; Dal Corso A.; Giannozzi, P. Phonons and related crystal properties from densityfunctional perturbation theory. Rev. Mod. Phys. 2001, 73, https://doi.org/10.1103/RevModPhys.73.515.

40. Mori-Sánchez, P.; Wu, Q.; Weitao Yang. Many-electron self-interaction error in approximate density functionals. J. Chem. Phys. 2003, 119, https://doi.org/10.1063/1.2403848.

41. de Boeij, M.P.L. van Leeuwen, R.; Berger, J.A.; Snijders, J.G. Ultra-nonlocality in Time-Dependent CurrentDensity-Functional Theory: Application to Conjugated Polymers. Phys. Rev. Lett. 2002, 88, https://doi.org/10.1103/PhysRevLett.88.186401. 